DFF 355/09/00

\title{
Parafermion Hall States from Coset Projections of Abelian Conformal Theories
}

\author{
Andrea CAPPELLI"I \\ I.N.F.N. and Dipartimento di Fisica, \\ Largo E. Fermi 2, I-50125 Firenze, Italy \\ Lachezar S. GEORGIEVII \\ Inst. Theoretische Physik, Universitat zu Köln, \\ Zülpicher Str. 77, 50937 Köln, Germany \\ Ivan T. TODOROVH \\ Institute for Nuclear Research and Nuclear Energy, \\ Tsarigradsko Chaussee 72, BG-1784 Sofia, Bulgaria
}

\begin{abstract}
The $\mathbb{Z}_{k}$-parafermion Hall state is an incompressible fluid of $k$-electron clusters generalizing the Pfaffian state of paired electrons. Extending our earlier analysis of the Pfaffian, we introduce two "parent" abelian Hall states which reduce to the parafermion state by projecting out some neutral degrees of freedom. The first abelian state is a generalized (331) state which describes clustering of $k$ distinguishable electrons and reproduces the parafermion state upon symmetrization over the electron coordinates. This description yields simple expressions for the quasi-particle wave functions of the parafermion state. The second abelian state is realized by a conformal theory with a $(2 k-1)$-dimensional chiral charge lattice and it reduces to the $\mathbb{Z}_{k}$-parafermion state via the coset construction $\widehat{s u(k)_{1}} \oplus \widehat{s u(k)_{1}} / \widehat{s u(k)_{2}}$. The detailed study of this construction provides us a complete account of the excitations of the parafermion Hall state, including the field identifications, the $\mathbb{Z}_{k}$ symmetry and the partition function.
\end{abstract}

September 2000

\footnotetext{
${ }^{*}$ E-mail: andrea.cappelli@fi.infn.it.

${ }^{\dagger}$ E-mail: lg@thp.uni-koeln.de. On leave of absence from I.N.R.N.E., Tsarigradsko Chaussee 72, BG-1784 Sofia, Bulgaria

†E-mail: todorov@inrne.bas.bg.
} 


\section{Introduction}

\subsection{Outline of the paper}

Conformal field theories have been successfully applied to describe the universal properties of quantum Hall states [1], such as symmetries, quantum numbers and low-energy dynamics of edge excitations [2]. The simplest Laughlin states with filling fraction $\nu=1,1 / 3,1 / 5, \ldots$ [3] are well understood in terms of abelian conformal theories with central charge $c=1$; most of our understanding of edge excitations, including their fractional statistics and dynamics, has been drawn from these states. Furthermore, the theoretical predictions have received important confirmations by experiments [四].

Recent studies have addressed more involved Hall states, such as that occurring at $\nu=5 / 2$. This plateau in the second Landau level has no analogue in the first level (i.e. at $\nu=1 / 2$ ); thus, it should be caused by some new dynamical mechanism. In Ref.[5], it was proposed that the electrons form pairs, in a way similar to BCS pairs in superconductors; these bosonic pairs can then form a Laughlin fluid with even denominator filling fraction. The ( $p$-wave) pairing of (spin-polarized) electrons is represented in the ground-state wave function by the Pfaffian term $\operatorname{Pf}\left(1 /\left(z_{i}-z_{j}\right)\right)$, which involves all possible pairs of electron coordinates. (Other states with different pairings were also considered [6] [2].)

There is a well-established relation between Hall states and conformal field theories, which allows to compute the filling fractions and the quantum numbers of the excitations; moreover, the analytic part of electron wave functions correspond to correlators of conformal fields. Following Ref. [5], the Pfaffian term can be reproduced by the correlator of Majorana fermions in the $c=1 / 2$ conformal field theory, i.e. the critical Ising model, plus the usual $c=1$ boson theory accounting for the charge of excitations.

It is remarkable that the Pfaffian Hall state possesses excitations with non-abelian fractional statistics [5]: the adiabatic transport of one excitation around another causes a multi-dimensional unitary transformation within a multiplet of degenerate wave functions, rather than a sign factor (Fermi statistics) or a phase (abelian anyon statistics). The non-abelian statistics is easily understood in the Ising conformal theory: the spin field $\sigma$ possesses the operator-product expansion $\sigma \cdot \sigma \sim \operatorname{Id}+\psi$, with two terms in the right hand side; therefore, multi-spin correlators expand into several terms (the conformal blocks), which transform among themselves under monodromy. Numerical analyses have shown that the Pfaffian state has a rather good overlap with the exact ground state at $\nu=5 / 2$ [7] ; therefore, there is an exciting possibility that new phenomena such as electron pairing and non-abelian statistics could be experimentally observed at sufficiently low temperatures for $2<\nu<3$.

\footnotetext{
$\S$ In contrast with the previous expectations favouring the Haldane-Rezayi paired state [6].
} 
Read and Rezayi have proposed [8] a generalization of the Pfaffian to a hierarchy of states in the second Landau level, which are described by the $\widehat{u(1)} \times \mathrm{PF}_{k}$ conformal theories, where $\mathrm{PF}_{k}$ stands for the $\mathbb{Z}_{k}$ parafermions [9]. These parafermion Hall states have filling fractions,

$$
\nu \equiv 2+\nu_{k}(M)=2+\frac{k}{k M+2}, \quad k=2,3, \ldots \quad M=1,3,5, \ldots,
$$

with the Pfaffian corresponding to the case $k=2$ and $M=1$. The same authors found that these Hall states have good overlap with the numerical exact ground states at $\nu=13 / 5,8 / 3$, i.e. for $M=1$ and $k=3,4$ \&n. Furthermore, Hall plateaus at these filling values have been experimentally observed by cooling down the sample at extremely low temperatures [10]. Read and Rezayi have shown that the $\mathbb{Z}_{k}$-parafermion Hall state possesses an interesting dynamics: the Hall fluid is made by clusters of $k$ electrons (generalizing the pairwise binding for $k=2$ ) and there are excitations with non-abelian statistics; other properties were discussed in Ref.[11].

This new Hall dynamics is expected to be universal, i.e. rather robust under small changes of the microscopic dynamics; therefore, it should be possible to describe it directly in the effective low-energy conformal field theory. For example, we would like to find conformal theory arguments for the quantum numbers of these Hall states and for the mechanism of clustering which actually yields the $\mathbb{Z}_{k}$ parafermions and non-abelian statistics.

Our general idea is to describe the non-abelian Hall states and the associated conformal theories by first introducing suitable parent abelian theories with same filling fraction and then by projecting down to the original non-abelian theoriest]. The projection preserves the filling fraction because it only affects the neutral degrees of freedom; moreover, it fulfills a number of consistency conditions to be specified later.

This two-step approach can be useful for disentangling the well-understood Hall physics of abelian states (such as the Laughlin fluids) from the new phenomena to be associated with the projection. The abelian states have a special status in the quantum Hall effect because they realize the $W_{1+\infty}$ symmetry, the natural symmetry of "simple" quantum incompressible fluids under area-preserving reparametrizations of the plane coordinates [13] 14. The $\mathbb{Z}_{k}$-parafermion states are only symmetric under the sub-algebra $\widehat{u(1)} \oplus \mathcal{W}_{k}$ [15] of the $W_{1+\infty}$ algebra; therefore, the abelian parent theories provide a framework for understanding this remarkable symmetry reduction, and the appearance of non-abelian statistics, as being the results of the projection.

In Ref. [16], we have already shown that the Pfaffian state can be described in

I See Ref.12 for a different attempt to describe non-abelian states with abelian degrees of freedom. 
terms of two abelian lattice theories by projecting out neutral degrees of freedom. Both parent abelian theories provide interesting descriptions of the Pfaffian physics; in particular, one projection relates the (331) with the Pfaffian states and describes the corresponding transition from the two-layer to the thick-layer $\nu=1 / 2$ Hall setups which was advocated in Ref. 17.

In this paper, we describe the generalization of these abelian descriptions to the parafermion states (see Ref. 18 for a preliminary account of this work). In Section 2, we generalize the (331) state to a $k$-component fluid of distinguishable electrons: one readily finds that this $c=k$ abelian theory has the correct filling fraction (1.1) and can be describe the $k$-electron clustering. Actually, $k$ electrons can meet at the same point, i.e. avoid the Pauli principle, by taking $k$ different "colours". We show that the conventional Laughlin wave functions of coloured electrons also describe the parafermion Hall states after the anti-symmetrization over all coordinates, which makes the electrons indistinguishable. We thus obtain simpler expressions for the known parafermion wave functions [8], as well as new expressions for the insertion of quasi-particles.

The anti-symmetrization of wave-function coordinates corresponds to a projection map between two conformal field theories with $c=k$ and $c=3 k /(k+2)$, respectively; besides the $k=2$ case $(c=2 \rightarrow 3 / 2)$ discussed before [16], this map has not been analysed in the literature. Therefore, this approach is not practical for a detailed description of the parafermion conformal theory. In Sections 3 and 4, we consider the better known description [19] of $\mathbb{Z}_{k}$ parafermions in terms of the coset construction 20]:

$$
\mathrm{PF}_{k}=\frac{\widehat{s u(k)_{1}} \oplus \widehat{s u(k)_{1}}}{\widehat{s u(k)_{2}}}, \quad c=\frac{2(k-1)}{k+2} .
$$

We remark that the numerator is actually a $c=2 k-2$ abelian theory; therefore, the parafermion Hall states can be described by a $c=2 k-1$ abelian state plus the coset projection. This second parent abelian theory (Section 3) possesses the extended affine symmetry $\widehat{u(1)} \oplus \widehat{s u(k)_{1}} \oplus \widehat{s u(k)_{1}}$; its charge lattice is maximally symmetric in the sense of Ref. [21], where it has been denoted by $\left(M+2 \mid{ }^{1} A_{k-1}{ }^{1} A_{k-1}\right)$. The two $s u(k)$ symmetries refer to quantum numbers which can be thought of as representing effective layer and iso-spin indices.

After the coset projection, described in Section 4 , there only remains the $\mathbb{Z}_{k}$ symmetry relative to the parafermion "charge" [9] as it should. It turns out that this charge is coupled to the fractional (anyon) part of the physical electric charge of the quasi-particles, as follows. The projection of neutral degrees of freedom in the $c=2 k-1$ abelian theory requires the decoupling of its charged and neutral sectors. This cannot be done globally within the maximally symmetric lattice, but give rise to a $\mathbb{Z}_{k}$ selection rule, relating the quasi-hole electric charge $(u(1)$ part) to the parafermion charge $\left(\mathrm{PF}_{k}\right.$ part $)$ modulo $k$. Therefore, the coset construction 
of the conformal theories for the parafermion Hall states uniquely determines the complete quantum numbers of the quasi-particle excitations; namely, it completely defines these Hall states starting from the original choice of maximally symmetric lattice. We explicitly construct the super-selection sectors (the irreducible representations) of these conformal theories, compute their characters and write their partition functions [22].

The description of quantum Hall states by coset conformal theories has also been independently proposed in Ref. [23]: here, we describe the detailed aspects of this construction for the parafermion Hall states, after having presented the main points in the preliminary note [18]. In agreement with the authors of Ref. 223, we believe that coset projections of abelian theories can be physically relevant, because the reduction of neutral degrees of freedom and of the central charge increases the stability of the Hall state (criterion (S1) in Ref. 23]). Let us also quote other projective constructions of non-abelian Hall states in the Refs. 24], which include the different coset $\mathrm{PF}_{k}=\widehat{s u(2)_{k}} / \widehat{u(1)}$.

In Section 5, we explicitly work out the coset construction of the $Z_{3}$-parafermion Hall state and analyze the properties of its quasi-particle excitations, their quantum numbers, character expressions and fusion rules. In the Conclusions, we briefly outline a set of postulates which could characterize rational conformal theories describing quantum Hall states, encompassing both abelian theories and coset projections.

\subsection{Abelian conformal theories and integer lattices}

We recall that an abelian theory is a rational conformal field theory with integer central charge $c=n$ and $\widehat{u(1)}^{n}$ affine symmetry; the current algebra is generated by $n$ abelian currents $J^{a}(z)=i \partial_{z} \phi^{a}(z)$, extended by vertex operators $Y(\underline{\lambda}, z)=$ : $\exp \left(i \lambda_{a} \phi^{a}(z)\right)$ :, such that their "charges" $\lambda_{a}$ are vectors of a $n$-dimensional lattice $\Gamma$. For example, the $\widehat{s u(k)_{1}}$ theory is the extension of $\widehat{u(1)}^{k-1}$ theory with the $A_{k-1}$ root lattice. In the quantum Hall effect, the lattices satisfy some specific physical conditions which are summarized hereafter. Following Ref. [21], we call chiral quantum Hall lattice an odd integral lattice associated with a charge vector $\underline{Q}$ of the dual lattice $\Gamma^{*}$, which assigns to any lattice point $\underline{q} \in \Gamma$ the electric

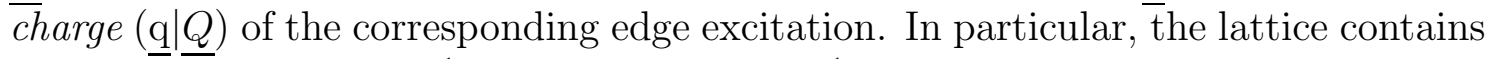
an electron excitation $\underline{q}^{1}$ with unit charge $\left(\underline{q}^{1} \mid \underline{Q}\right)=-1$. Furthermore, the norm $|\underline{q}|^{2}=(\underline{q} \mid \underline{q})$ of the vectors $\underline{q} \in \Gamma$ yields twice the conformal dimensions, i.e. twice the spin of the corresponding excitation, while the scalar product $(\underline{q} \mid \underline{p})$ is the relative statistics of the excitations $\underline{q}$ and $\underline{p}$.

The charge vector $\underline{Q}$ satisfies the following conditions:

(i) it is primitive, i.e., not a multiple of any other vector $\underline{q}^{*} \in \Gamma^{*}$; 
(ii) it is related to $\nu$ by:

$$
|\underline{Q}|^{2}=\nu ;
$$

(iii) it obeys the charge-statistics relation for boson/fermion excitations:

$$
(\underline{Q} \mid \underline{q})=|\underline{q}|^{2} \quad \bmod 2, \quad \text { for any } \underline{q} \in \Gamma .
$$

Remark. For Hall states in the second Landau level, $2<\nu \leq 3$, such as those in Eq.(11.1), one should actually replace $\nu \rightarrow(\nu-2)$ in (1.3), because the completely filled first level is silent but adds two to the filling fraction.

The non-equivalent quasi-particles in the Hall state correspond to the irreducible representations of the extended algebra and are labelled by the points $\underline{\lambda} \in \Gamma^{*} / \Gamma$ where $\Gamma^{*}$ is the dual latticel. The low-dimensional chiral Hall lattices have been completely classified in Ref. [21].

\subsection{Projective descriptions of the Pfaffian state}

The $\nu=2+1 / 2$ Pfaffian Hall state [5] corresponds to the $c=3 / 2$ conformal theory of one $u(1)$ current and the Ising model (i.e. the Majorana fermion). The two parts describe corresponding pieces in the ground-state wave function (for even $N$ ):

$$
\Psi\left(z_{1}, \ldots, z_{N}\right)=\operatorname{Pf}\left(\frac{1}{z_{i}-z_{j}}\right) \prod_{1=i<j}^{N}\left(z_{i}-z_{j}\right)^{2}
$$

namely, the $N$-point function of the Majorana fermions produces the Pfaffian and the vertex-operator correlator yields the usual Laughlin factor.

In Ref. [16] we showed that the Pfaffian state could be realized as a two-step projection of a lattice abelian theory. The starting point was a $c=3$ theory whose Hall lattice $\Gamma$ is maximally symmetric [21] (this definition is recalled in Section 3); its Gram matrix $\left(G_{\Gamma}\right)_{i j}=\left(\underline{\mathrm{q}}^{i} \mid \underline{q}^{j}\right)$ has the following form网, in the basis spanned by the electron charge $\underline{q}^{1}$ and two neutral vectors:

$$
G_{\Gamma}=\left[\begin{array}{rrr}
3 & 1 & -1 \\
1 & 2 & 0 \\
-1 & 0 & 2
\end{array}\right], \quad Q=(1,0,0) \in \Gamma^{*} .
$$

In this equation, we also wrote the components of the charge vector $Q$ in the dual basis. The neutral sub-lattice of $\Gamma$ (i.e., the one orthogonal to $\underline{Q}$ ) is actually the

\footnotetext{
$\|$ We recall that $\Gamma^{*} / \Gamma$ is a finite abelian group of order $(\operatorname{det} \Gamma)$ whose multiplication law represents the fusion rules.

** The sign convention for the matrix elements $\left(G_{\Gamma}\right)_{31}=\left(G_{\Gamma}\right)_{13}$ differs from that of Ref. [16] as explained in Section 3.
} 
direct sum of two root lattices for the $s u(2)$ Lie algebra and therefore the abelian conformal theory possesses the extended $\widehat{u(1)} \oplus \widehat{s u(2)_{1}} \oplus \widehat{s u(2)_{1}}$ affine algebra.

Since the Gram matrix (1.6) is not decomposable, the neutral degrees of freedom are not completely decoupled from the charge; the isospin-charge separation is necessary for the intended projections of neutral degrees of freedom and can be achieved in the finer orthogonal lattice $\Gamma_{2,1,1}$ : the points of the original lattice $\Gamma$ correspond to those points in $\Gamma_{2,1,1}$ which satisfy $(-1)^{n_{1}+n_{2}+n_{3}}=1$ where $n_{i} \in \mathbb{Z}$ are the components in the basis of $\Gamma_{2,1,1}$. This leads to the $\mathbb{Z}_{2}$ selection rule, the "parity rule", which couples the independent neutral and charged sectors of $\Gamma_{2,1,1}$ :

$$
\Gamma=\Gamma_{2,1,1} / \mathbb{Z}_{2}, \quad G_{\Gamma_{2,1,1}}=\left[\begin{array}{lll}
2 & 0 & 0 \\
0 & 1 & 0 \\
0 & 0 & 1
\end{array}\right] .
$$

Note that the rank-two neutral sub-lattice of $\Gamma_{2,1,1}$ corresponds to the $c=2$ conformal theory of two Weyl fermions which do not carry physical charge because the corresponding vectors are orthogonal to $\underline{Q}$ (1.6).

The Majorana fermion describing the Pfaffian state is obtained by two successive projections [16] which preserves the filling fraction and the conformal dimension of the electron field so as to maintain the spin-statistics relation:

(i) Project out the second Weyl fermion corresponding to the third basis vector of $\Gamma_{2,1,1}$. This reduces the Virasoro central charge by 1 but preserves the topological order,i.e., the number of irreducible representations, given by the determinant of $G_{\Gamma}$ [2]. Taking into account the $\mathbb{Z}_{2}$ parity rule (inherited from the maximally symmetric state) this projection gives rise to the $331 \mathrm{Hall}$ state. In more standard notations [2], corresponding to $Q=(1,1)$, its Gram matrix is $\left(\begin{array}{ll}3 & 1 \\ 1 & 3\end{array}\right)$ or more generally $\left(\begin{array}{ll}m+1 & m-1 \\ m-1 & m+1\end{array}\right)$ (see Section 2).

(ii) Project out the imaginary part of the first Weyl fermion (i.e., one Majorana fermion) corresponding to the second basis vector of $\Gamma_{2,1,1}$. This neutral projection removes $1 / 2$ from the central charge and also decreases the topological order. The resulting Pfaffian state is made by the remaining Majorana fermion, the abelian current in the first component and the $\mathbb{Z}_{2}$ parity rule.

The latter map between the (331) state and the Pfaffian could occur in a doublelayer Hall device at $\nu=1 / 2$ [17], and represent the transition between a regime of low tunneling between the layers (distinguishable electrons, (331) state) to the high-tunneling limit (indistinguishable, i.e. one-species electrons, Pfaffian state) [16].

The combination of the two projections amounts to gauging out the tensor product,

$$
\operatorname{Weyl}\left(\Psi^{*}, \Psi\right) \otimes \operatorname{Ising}(\varphi)=\widehat{s u(2)_{2}},
$$


where the $\widehat{s u(2)}$ currents are identified with:

$$
H(z)=2: \Psi^{*}(z) \Psi(z):, \quad E^{+}(z)=\sqrt{2} \varphi(z) \Psi^{*}(z), \quad E^{-}(z)=\sqrt{2} \varphi(z) \Psi(z) .
$$

Finally, taking into account the fact that the neutral sub-algebra is originally $\widehat{s u(2)} \oplus$ $\widehat{s u(2)_{1}}$, these two steps can be seen as realizing the coset construction (1.2) for $k=2$ in the neutral part of the theory. To combine it to the electric part we use the fact that the full coset theory inherits the $\mathbb{Z}_{2}$ parity rule which is preserved by both steps of the projection.

\subsection{The parafermion Hall states and electron clustering}

The Pfaffian state describes the incompressible Hall fluid of (spin polarized) electron pairs; actually, the Pfaffian term in the wave function has been explicitly derived as the long-range limit of a BCS-like wave function [25]. An effective interaction which allows such pairing is given by a three-body pseudo-potential, for which the Pfaffian wave function (1.5) is the exact zero-energy eigenstate with lowest angular momentum [5].

The $\mathbb{Z}_{k}$-parafermion Hall states introduced in Ref. [8] are the generalizations to incompressible fluids made by clusters of $k$ electrons; the corresponding $(k+1)$-body interaction is:

$$
H=v \sum_{1 \leq i_{1}<i_{2}<\ldots<i_{k+1}}^{N} \delta^{2}\left(z_{i_{1}}-z_{i_{2}}\right) \delta^{2}\left(z_{i_{2}}-z_{i_{3}}\right) \cdots \delta^{2}\left(z_{i_{k}}-z_{i_{k+1}}\right)
$$

The $\mathbb{Z}_{k}$-parafermion wave functions are zero-energy states which vanish when $(k+1)$ electrons meet together; the ground-state solution of lowest angular momentum: $(i)$ should not vanish when $k$ electron meet; and (ii) should go to zero as weakly as possible when a further electron is approaching. The property $(i)$ indicates that $k$ electrons have formed a bound state (a cluster), and it is certainly not satisfied by an ordinary Hall state of (unbound) electrons like the Laughlin states.

As nicely discussed in Ref. [11], these two properties imply the following factorization of the ground-state wave function, when $k$ coordinates are set equal (say $\left.\left\{z_{1}, z_{2}, \ldots, z_{k}\right\}\right)$ :

$$
\Psi_{k}\left(z_{k}, z_{k}, \ldots, z_{k}, z_{k+1}, \ldots, z_{N}\right) \propto \prod_{i=k+1}^{N}\left(z_{k}-z_{i}\right)^{2} \Psi_{k}\left(z_{k+1}, z_{k+2}, \cdots, z_{N}\right)
$$

The right hand side of this equation contains the same wave function for $(N-k)$ electrons. The vanishing behaviour $\left(z_{k}-z_{k+1}\right)^{2}$ is the lowest one allowed by Bose statistics, which is assumed in (1.9); actually, $\Psi_{k}$ is the reduced wave function, 


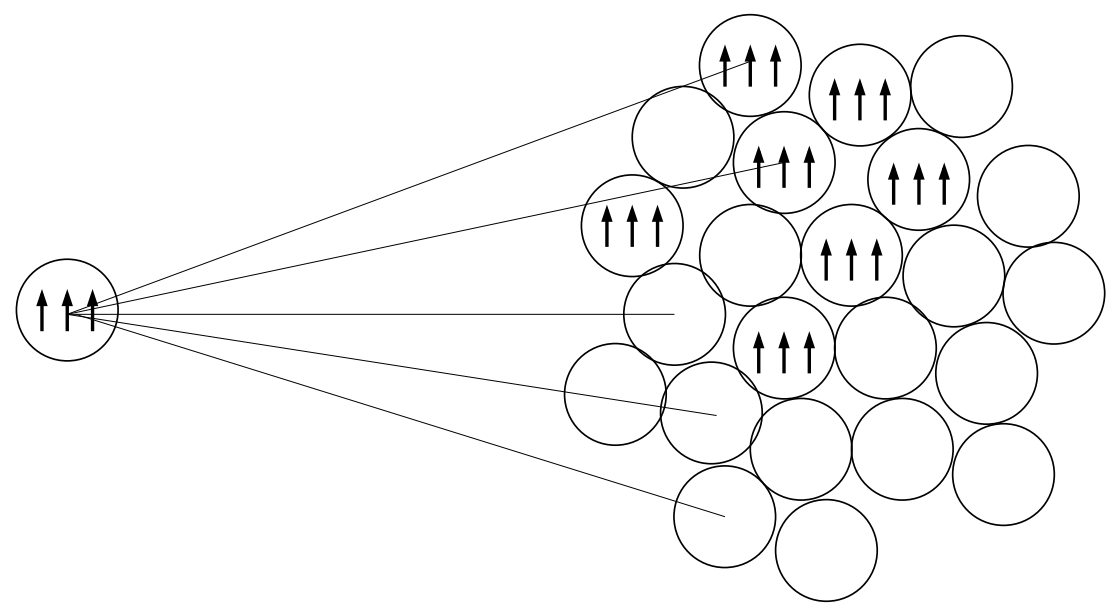

Figure 1: Factorization of one cluster in the parafermion Hall state of three-electron clusters, as described by Eq.(1.9).

which is obtained from the complete one describing the fillings (1.1) by stripping out a standard antisymmetric factor $\prod_{i>k}\left(z_{k}-z_{i}\right)^{M}$, with $M$ odd. (Equivalently, the reduced wave function (1.9) describes the (unphysical) bosonic parafermion state with $M=0)$. Therefore, the two properties $(i),(i i)$ of the $k$-cluster Hall fluids are accounted for by Eq.(1.9).

The factorization equation (1.9) should be interpreted as the analogue of the cluster decomposition theorem for statistical models (see Figure (11)); in the quantum Hall effect, there cannot be complete factorization for distant sub-systems, owing to the long-range order: this explains the factor $\prod_{k<i}\left(z_{k}-z_{i}\right)^{2}$. Another interpretation is that the $k$-electron cluster, once formed at $z=z_{k}$, interacts with a further electron at, say, $z=z_{k+1}$, as an ordinary particle of a Laughlin fluid.

The Pfaffian wave function (1.5) $(M=1, k=2)$ divided by $\prod_{k<i}\left(z_{k}-z_{i}\right)$ does satisfy the factorization equation (1.9). For general $k$, the form of $\Psi_{k}$ is considerably more involved and was obtained in Ref.[B] as follows; one first introduces a polynomial of $2 k$ variables, corresponding to the two clusters $\left\{z_{1}, \ldots, z_{k}\right\}$ and $\left\{z_{k+1}, \ldots, z_{2 k}\right\}$ :

$$
\begin{aligned}
\chi_{1,2}\left(z_{1}, \cdots, z_{k} ; z_{k+1}, \ldots, z_{2 k}\right)= & \left(z_{1}-z_{k+1}\right)\left(z_{1}-z_{k+2}\right)\left(z_{2}-z_{k+2}\right)\left(z_{2}-z_{k+3}\right) \cdots \\
& \cdots\left(z_{k}-z_{2 k}\right)\left(z_{k}-z_{k+1}\right) .
\end{aligned}
$$

(Note that these factors can be graphically represented by links forming a closed $2 k$-polygon.) Next, the wave function exists for $N=k n$ electrons, which are divided in $n$ clusters labelled by the index $r, 0 \leq r<n$, and reads:

$$
\begin{aligned}
\Psi_{k} & \left(z_{1}, \ldots, z_{k}, \ldots, z_{k r+1}, \ldots, z_{k r+k}, \ldots, z_{(n-1) k+1}, \ldots, z_{k n}\right) \\
= & \mathcal{S}_{N}\left[\prod_{0 \leq r<s<n} \chi_{r, s}\left(z_{k r+1}, \ldots, z_{k r+k} ; z_{k s+1}, \ldots, z_{k s+k}\right)\right], \quad(N=n k) .
\end{aligned}
$$


Here, $\mathcal{S}_{N}$ represents the complete symmetrization over the $N$ coordinates of the expression inside the square brackets, and is properly normalized to be a projector: $\mathcal{S}_{N}^{2}=\mathcal{S}_{N}$

The relation of this wave function to a $N$-point function of the $\mathbb{Z}_{k}$-parafermion conformal theory was also found in Ref. [8] and fully proved in Ref. [11]. It reads:

$$
\Psi_{k}\left(z_{1}, \ldots, z_{N}\right) \propto\left\langle\psi_{1}\left(z_{1}\right) \psi_{1}\left(z_{2}\right) \cdots \psi_{1}\left(z_{N}\right)\right\rangle \prod_{1=i<j=N}\left(z_{i}-z_{j}\right)^{2 / k},
$$

where $\psi_{1}(z)$ is the first of the chiral parafermion fields $\psi_{\ell}(z)$ with conformal dimension $\Delta_{\ell}$ [9]:

$$
\psi_{\ell}(z), \quad \ell \bmod k, \quad\left(\psi_{0} \equiv I\right), \quad \Delta_{\ell}=\frac{\ell(k-\ell)}{k} .
$$

In order to prove the equivalence of the expressions (1.11) and (1.12), one first observes that they are entire functions with equal asymptotic behaviour (same total angular momentum); then one compares their behaviour when $(k+1)$ electrons meet together [11]. In the case of (1.12), one can use the operator-product expansions [9]:

$$
\begin{aligned}
\psi_{\ell}(z) \psi_{\ell^{\prime}}(0) & =z^{-2 \ell \ell^{\prime} / k} \psi_{\ell+\ell^{\prime}}(0)+\cdots, \quad 0<\ell+\ell^{\prime}<k ; \\
\psi_{\ell}(z) \psi_{k-\ell}(0) & =z^{-2 \ell(k-\ell) / k}\left(I+z^{2} \frac{2 \Delta_{\ell}}{c} T(0)+\cdots\right) .
\end{aligned}
$$

Since the fusion of $k$ fields $\psi_{1}$ yields the identity operator, the correlator (1.12) does not vanish when $k$ electrons meet at the same point, but becomes the same expression for the remaining $(N-k)$ coordinates; this checks the factorization formula (1.9), which is also satisfied by the other form (1.11) [8]. The identity of the two wave functions is finally proved [11] by checking that there is no linear sub-leading term in the expansion of (1.11) in $\left(z_{k-1}-z_{k}\right)$, at $z_{1}=z_{2}=\cdots=z_{k-1}$, since this term does not appear in operator product (1.15) either; such linear term would have implied the presence of another $\widehat{u(1)}$ current in the corresponding conformal theory, which is absent in the $\mathbb{Z}_{k}$-parafermion theory [ []].

The $\mathbb{Z}_{k}$-parafermion conformal theory also describes the excitations of this Hall state, which are created by its primary conformal fields (multiplied by appropriate choices of vertex operators for the $\widehat{u(1)}$ part); besides the $\psi_{\ell}$, with abelian fusion rules and statistics, there exist the spin fields $\sigma_{\ell}$ with dimensions $d_{\ell}$ :

$$
\sigma_{\ell}(z), \quad \ell=1, \ldots, k-1, \quad d_{\ell}=\frac{\ell(k-\ell)}{2 k(k+2)} .
$$

In particular, the first field $\sigma_{1}$ creates the elementary quasi-hole and obeys nonabelian statistics as in the Pfaffian case (see Section 5 for examples).

The corresponding wave functions can be written as correlators of the spin fields inserted in the electron wave function; however, conformal-theory methods do not 
immediately give explicit many-particle expressions; in Ref. [8], the simplest case of $k$ quasi-particle wave function, i.e. $\left\langle\sigma_{1}\left(\eta_{1}\right) \cdots \sigma_{1}\left(\eta_{k}\right) \psi_{1}\left(z_{1}\right) \cdots \psi_{1}\left(z_{N}\right)\right\rangle$, was written down. In the next Section, we shall obtain rather explicit expressions for all quasiparticle wave functions by using an abelian conformal theory and a projection map to the parafermion theory.

\section{Generalized (331) abelian theory and parafermion wave functions}

\subsection{Ground-state wave function}

The generalization of the (331) abelian theory (recalled in Section 1.2) is a $c=k$ abelian theory with integer $k$-dimensional lattice $\tilde{\Gamma}$ specified by the following Gram matrix and charge vector:

$$
\begin{aligned}
\left(G_{\tilde{\Gamma}}\right)_{i j} & = \begin{cases}M+2 & i=j=1, \ldots, k, \\
M & i \neq j\end{cases} \\
\underline{Q} & =(1,1, \ldots, 1) .
\end{aligned}
$$

Form the Gram matrix, also called " $K$-matrix" [2] in the basis (2.1), we obtain the following filling fractions,

$$
\nu_{k}=\sum_{i, j=1}^{k}\left(G_{\tilde{\Gamma}}\right)_{i j}^{-1}=\frac{k}{k M+2}
$$

which reproduce the values of the parafermion states (1.1). The abelian conformal theory is meant to describe the Hall state of $k$ different species of electrons, which are characterized by another quantum number conventionally called "colour". There are $k$ different sets of coordinates, $\left\{z_{i}^{(a)}\right\}, a=1, \ldots, k$, because the different species are distinguishable and anti-symmetrization is done within each set only. Each

species corresponds to a lattice axis in the basis (2.1), and all species have the same spectrum of excitations due to the permutation symmetry of $G_{\tilde{\Gamma}}$.

The ground state wave function is the expectation value of $k$-component vertex operators, whose conformal dimensions can be read off from the $K$ matrix [2]; the result is, for $N=n k$ electrons:

$$
\begin{aligned}
\Psi_{\tilde{\Gamma}}\left(z_{1}^{(1)}, \ldots, z_{n}^{(1)} ; \cdots ; z_{1}^{(k)}, \ldots, z_{n}^{(k)}\right) & =\left[\prod_{1 \leq a<b \leq k} \prod_{1 \leq i<j \leq n}\left(z_{i}^{(a)}-z_{j}^{(b)}\right)^{M}\right] \\
& \times \prod_{a=1}^{k} \prod_{1 \leq i<j \leq n}\left(z_{i}^{(a)}-z_{j}^{(a)}\right)^{2} .
\end{aligned}
$$


In order to compare this expression with the reduced $(M=0)$ wave function of the parafermion Hall states in the previous Section, we shall hereafter discard the piece between square brackets in the r.h.s of (2.3).

It turns out that $\left.\Psi_{\tilde{\Gamma}}\right|_{M=0}$ satisfies the two properties for the $k$-electron cluster Hall fluid discussed in the previous Section: (i) it does not vanish when $k$ electrons meet at the same point (provided that they are taken of different colours), and (ii) it vanishes as $\left(z_{k}-z_{k+1}\right)^{2}$ when a further electron is added. The first point follows from the fact that distinguishable electrons do not satisfy the Pauli exclusion principle; the second property is manifest in (2.3). Note also that the abelian (2.3) and the parafermion (1.12) ground states possess the same total angular momentum. We conclude that the abelian Hall state (2.3) describes the incompressible fluid of clusters of $k$ distinguishable electrons with the filling fractions (1.1).

The next step is to describe the case of indistinguishable electrons; this is achieved by first renaming the coordinates $\left\{z_{i}^{(a)}\right\}$ in $(2.3)$ into a single set:

$$
\mathcal{R}: \quad z_{j}^{(\ell)} \longrightarrow z_{(j-1) k+\ell}, \quad j=1, \ldots, n, \quad \ell=1, \ldots, k, \quad N=k n,
$$

for example $\left\{z_{1}^{(1)}, \ldots, z_{1}^{(k)}\right\}=\left\{z_{1}, \ldots, z_{k}\right\}, \quad\left\{z_{2}^{(1)}, \ldots, z_{2}^{(k)}\right\}=\left\{z_{k+1}, \ldots, z_{2 k}\right\}$; next, the wave function is symmetrized over all the $N$ coordinates $\left\{z_{j}\right\}$. In conclusion, we propose the following "abelian" expression for the $\mathbb{Z}_{k}$-parafermion ground state (1.12):

$$
\widetilde{\Psi}_{k}\left(z_{1}, \ldots, z_{N}\right)=\left.\widetilde{\mathcal{S}}_{N}\left[\prod_{a=1}^{k} \prod_{1 \leq i<j \leq n}\left(z_{i}^{(a)}-z_{j}^{(a)}\right)^{2}\right]\right|_{\mathcal{R}}, \quad(N=n k)
$$

where $\widetilde{\mathcal{S}}_{N}=\left(\widetilde{\mathcal{S}}_{N}\right)^{2}$ implements again the full symmetrization and is normalized as a projector. This expression compares well with the form (1.11): it looks familiar of standard Laughlin fluids and the physics of $k$-clustering is hidden in the projection made by $\widetilde{\mathcal{S}}_{N}$; let us now prove that $(2.5)$ is another form of the parafermion groundstate wave function (1.12).

Proof. We follow the same steps of the previous argument of the equivalence of the wave functions (1.11) and (1.12). We first argue that (2.5) satisfies the factorization (1.9); we rewrite (2.5) and consider the limit $z_{1} \sim z_{2} \sim \cdots \sim z_{k}$ :

$$
\begin{aligned}
\widetilde{\Psi}_{k} & \left(z_{1}, z_{2} \ldots, z_{k} ; z_{k+1}, \ldots, z_{N}\right) \\
= & \left.\widetilde{\mathcal{S}}_{N}\left[\prod_{a=1}^{k} \prod_{j=2}^{n}\left(z_{1}^{(a)}-z_{j}^{(a)}\right)^{2} \prod_{a=1}^{k} \prod_{2 \leq i<j \leq n}\left(z_{i}^{(a)}-z_{j}^{(a)}\right)^{2}\right]\right|_{\mathcal{R} ; z_{1}=z_{2}=\cdots=z_{k}} \\
\propto & \left.\widetilde{\mathcal{S}}_{N-k}\left[\prod_{a=1}^{k} \prod_{j=2}^{n}\left(z_{k}-z_{j}^{(a)}\right)^{2} \prod_{a=1}^{k} \prod_{2 \leq i<j \leq n}\left(z_{i}^{(a)}-z_{j}^{(a)}\right)^{2}\right]\right|_{\mathcal{R}}
\end{aligned}
$$


We analyse the various terms in the r.h.s. of (2.6) which are produced by the symmetrization over the coordinates; there are permutations acting within the first set of $k$ variables $I=\left\{z_{1}, \ldots, z_{k}\right\}$ (called $\sigma^{\prime} \in \mathcal{S}_{k}$ ), those within the rest of the variables $\bar{I}=\left\{z_{k+1}, \ldots, z_{N}\right\}$ (called $\left.\sigma \in \mathcal{S}_{N-k}\right)$, and between the two sets $\left(\tau \in \mathcal{S}_{N}\right)$. The identity permutation identifies $z_{1}^{(a)}=z_{k}, a=1, \ldots, k$, and yields a non-vanishing contribution, because there is no factor $\left(z_{i}-z_{j}\right)^{2}$ connecting pairs of collapsing coordinates (i.e. there are no "links" among them and these coordinates belong to a "cluster", in the language of Ref. [8]). The $\sigma^{\prime}$ permutations produce just a multiplicity for the same contribution; the $\tau$ permutations exchange one coordinate in $I$ with one coordinate in $\bar{I}$; the form of the wave function is such that this exchange necessarily introduces a link between two variables in $I$ and the corresponding contribution to the wave function vanishes quadratically at $z_{1}=\ldots=z_{k}$.

Therefore, all non-vanishing terms of $\widetilde{\Psi}_{k}$ in the limit $z_{1} \sim z_{2} \sim \cdots \sim z_{k}$ are accounted for by the permutations $\sigma \in \mathcal{S}_{N-k}$, leading to Eq. (2.7); next, we observe that the first factor in the r.h.s. of (2.7) is already symmetric over the remaining $(N-k)$ variables, thus, it can be pulled out of the symmetrization, and becomes the correct Laughlin factor in the decomposition (1.9); the remaining expression inside the square brackets is $\widetilde{\Psi}_{k}$ for $(N-k)$ electrons, up to an overall factor. This completes the proof that the wave function (2.5) satisfies the decomposition (1.9).

Next, we proceed to check that the linear sub-leading term in the operatorproduct expansion (1.15) is also absent in the expansion of (2.5): following Ref. [11, this amounts to show that, after taking $(k-1)$ variables equal, $z_{1}=z_{2}=\cdots=z_{k-1}$, the expansion in $\left(z_{k-1}-z_{k}\right)$ takes the following form to leading order (see Eq.(44) of Ref.[1]):

$$
\begin{aligned}
\widetilde{\Psi}_{k} & \left(z_{k-1}, z_{k-1}, \ldots, z_{k-1}, z_{k}, z_{k+1}, \ldots, z_{N}\right) \\
& \propto\left[\left(1+\frac{2(k-1)}{k}\left(z_{k-1}-z_{k}\right) \sum_{i>k}^{N} \frac{1}{z_{k}-z_{i}}\right) \prod_{i>k}^{N}\left(z_{k}-z_{i}\right)^{2}\right] \widetilde{\Psi}_{k}\left(z_{k+1}, \ldots, z_{N}\right)(2 .
\end{aligned}
$$

In order to analyse the different terms generated by the permutations in $\widetilde{\Psi}_{k}(2.5)$, we now divide the coordinates into three sets, $I=\left\{z_{1}, z_{2}, \ldots, z_{k-1}\right\}, I^{\prime}=\left\{z_{k}\right\}$ and $\bar{I}=\left\{z_{k+1}, \ldots, z_{N}\right\}$. Again, the (non-trivial) permutations acting between $(I \leftrightarrow \bar{I})$ introduce a link between two coordinates in $I$ and give a vanishing contribution to (2.8); the permutations acting between $\left(I^{\prime} \leftrightarrow \bar{I}\right)$ also introduce a link, which is $\left(z_{k-1}-z_{k}\right)^{2}$ and thus subleading to (2.8). Therefore, one is left with the permutations of $\mathcal{S}_{N-k}$ acting within $\bar{I}$, and the permutations between $\left(I \leftrightarrow I^{\prime}\right)$; a factorization is possible similar to $(2.7)$, with a term corresponding to $\widetilde{\Psi}_{k}\left(z_{k+1}, \ldots, z_{N}\right)$ and a factor of the form:

$\prod_{\left\{i_{1}, \ldots, i_{k-1}, i_{k}\right\}}\left(z_{k-1}-z_{i_{1}}\right)^{2} \cdots\left(z_{k-1}-z_{i_{k-1}}\right)^{2}\left(z_{k}-z_{i_{k}}\right)^{2}+$ permutations of $\left(i_{1}, i_{2}, \ldots, i_{k}\right)$. 


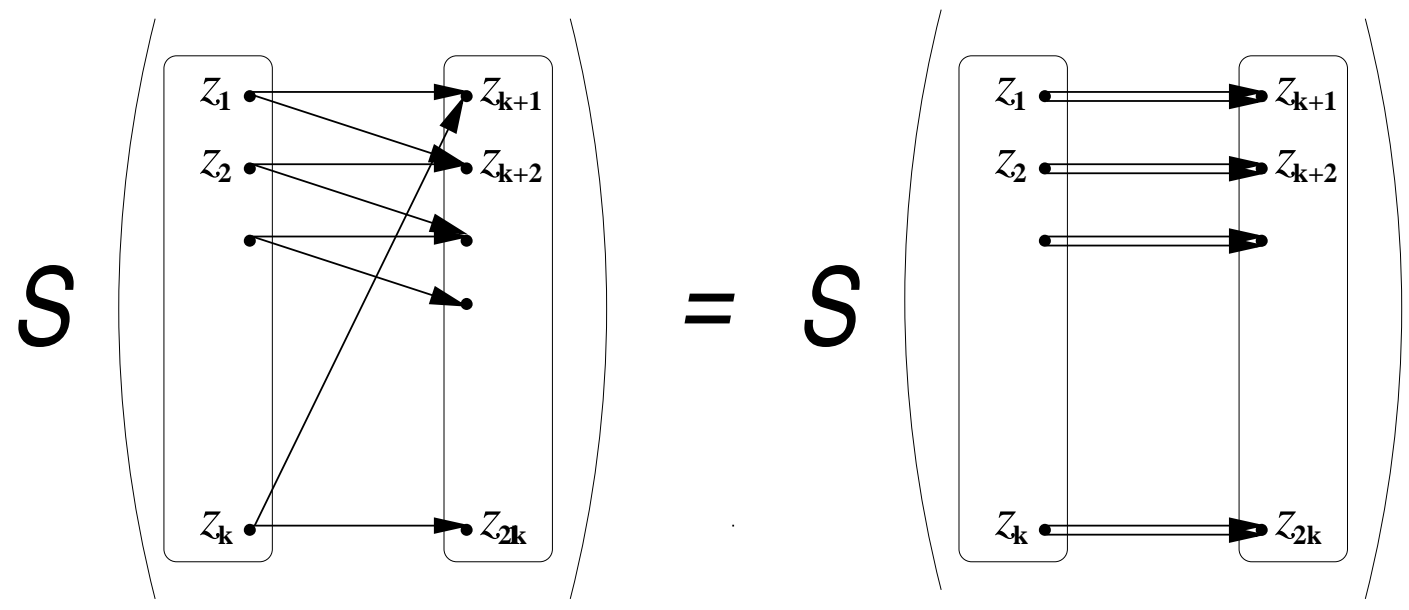

Figure 2: Equivalence of clusters interactions for the $\mathbb{Z}_{k}$-parafermion wave functions: under coordinate symmetrization, one can deviate some of the links $\left(z_{i}-z_{j}\right)$ (represented by arrows) connecting electrons of different clusters.

The expansion of this expression to order $O\left(z_{k-1}-z_{k}\right)$ matches the form within square brackets in Eq.(2.8); this completes the proof that (2.5) is an equivalent form for the parafermion ground-state wave function (1.11). This equivalence can be depicted as in Figure 2, in the case of the two clusters $\left\{z_{1}, \ldots, z_{k}\right\}$ and $\left\{z_{k+1}, \ldots, z_{2 k}\right\}$ connected by the links $\left(z_{i}-z_{j}\right)$.

\subsection{Quasi-particle wave functions and the origin of non- abelian statistics}

The strategy for writing quasi-particle wave functions of the parafermion Hall states is now rather clear; we use standard Laughlin quasi-particle wave functions in the abelian theory of distinguishable electrons and then apply the symmetrization over all coordinates to get the wave functions of the parafermion theory. From conformal field theory, we know that the number of quasi-particle excitations should be a multiple of $k$ in the $\mathbb{Z}_{k}$ theory so that the wave functions are local in the electron coordinates. For $k$ quasi-holes at positions $\left\{\eta_{1}, \ldots, \eta_{k}\right\}$, we can write:

$$
\begin{aligned}
& \Psi_{k}\left(\eta_{1}, \ldots, \eta_{k} ; z_{1}, \ldots, z_{N}\right)= \\
& \left.\widetilde{\mathcal{S}}_{N}\left[\prod_{a=1}^{k} \prod_{i=1}^{n}\left(\eta_{a}-z_{i}^{(a)}\right) \prod_{a=1}^{k} \prod_{1 \leq i<j \leq n}\left(z_{i}^{(a)}-z_{j}^{(a)}\right)^{2}\right]\right|_{\mathcal{R}}, \quad(N=n k) .
\end{aligned}
$$

This expression can be shown to be equivalent to that proposed in Ref. [8], by using the same arguments as before. Note that originally coloured electrons imply coloured quasi-holes, and thus there is a unique way to write a wave function with colour matching. 
For $m k$ quasi-holes, $m=2,3, \ldots$, we can instead write several expressions, depending on how the quasi-holes are divided into clusters of $k$ members. For $2 k$ quasiholes, we can e.g. split the coordinates $\left\{\eta_{1}, \ldots, \eta_{2 k}\right\}$ into $(1,2, \ldots, k)(k+1, \ldots, 2 k)$ and obtain the wave function:

$$
\begin{aligned}
\Psi_{k} & \left(\eta_{1}, \ldots, \eta_{k} ; \eta_{k+1}, \ldots, \eta_{2 k} ; z_{1}, \ldots, z_{N}\right) \\
= & \left.\widetilde{\mathcal{S}}_{N}\left[\prod_{a=1}^{k} \prod_{i=1}^{n}\left(\eta_{a}-z_{i}^{(a)}\right)\left(\eta_{k+a}-z_{i}^{(a)}\right) \prod_{a=1}^{k} \prod_{1 \leq i<j \leq n}\left(z_{i}^{(a)}-z_{j}^{(a)}\right)^{2}\right]\right|_{\mathcal{R}}
\end{aligned}
$$

Analogous expressions can be written for the other $2 k ! / 2(k !)^{2}$ choices of ordering; however, the number of independent wave functions is much lower, because there are non-trivial linear dependences among these polynomial expressions. The actual independent quasi-particle states are better given by the corresponding conformal blocks of the $\mathbb{Z}_{k}$-parafermion conformal theory. Their number is computable using the fusion rules of the fields $\sigma_{1}$ with itself and with $\psi_{1}$ : for $k=2$, there are $2^{m-1}$ independent $2 m$ quasi-hole wave functions [5], and for $k=3$ their multiplicities are given the Fibonacci number $F_{3 m-2}$ [11.

These results have been already found for the Pfaffian state in Ref. 26]: as an example, we recall the case of the three possible 4 quasi-hole wave functions of the Pfaffian state, with coordinate splittings: (12)(34), (13)(24) and (14)(23) (see Eq. (2.10)); only two of them are linearly independent due to the identity $\left(z_{i j} \equiv z_{i}-z_{j}\right.$, $\left.\eta_{i j} \equiv \eta_{i}-\eta_{j}\right)$ :

$$
z_{12} z_{34} \eta_{12} \eta_{34}+z_{13} z_{42} \eta_{13} \eta_{42}+z_{14} z_{23} \eta_{14} \eta_{23}=0
$$

The phenomena of: i) multiplicities of quasi-particle states for given positions and quantum numbers, and ii) linear dependences in the natural basis with simple monodromy transformations (cf. (2.10)), have been shown in Ref. [26] to characterize the non-abelian statistics of the Pfaffian quasi-holes; here, we can extend their analysis to any $k$. Let us rephrase the argument of Ref.[26]: the exchange of, say, two quasi-particles at positions $\left(\eta_{1}, \eta_{k+1}\right)$, would map (2.10) into the analogous expression for the coordinate splitting: $(k+1,2,3, \ldots, k)(1, k+2, k+3, \ldots, 2 k)$; if all the wave functions $\left(i_{1}, \ldots, i_{k}\right)\left(i_{k+1}, \ldots, i_{2 k}\right)$ were linear independent, these exchange transformations could be simultaneously diagonalized and would amount to multiplications by a phase, i.e. to abelian anyon statistics. However, the wave functions form a degenerate basis, such that the exchanges are represented by nontrivial (non-diagonalizable) matrices on the basis of independent functions, leading to non-abelian statistics.

These two options are exemplified by the quasi-particle wave functions of the parafermion Hall state (2.10) and of the parent abelian state (namely, Eq.(2.10) without $\widetilde{\mathcal{S}}_{N}$ ), respectively:

$$
\begin{array}{ll}
\text { no projection } & \rightarrow \text { independent basis } \rightarrow \text { abelian statistics }, \\
\text { projection } & \rightarrow \text { degenerate basis } \rightarrow \text { non - abelian statistics. }
\end{array}
$$


In conclusion, we have shown that the origin of non-abelian statistics can be simply and quite generally understood as the effect of the projection from a parent abelian Hall state; without the reference to an abelian state, the non-abelian statistics would have seemed to be the consequence of rather mysterious algebraic properties of specific wave functions [26]. The other side of the story is that the projection also breaks the generic $W_{1+\infty}$ symmetry of abelian Hall states 13 down to the $\mathcal{W}_{k}$ symmetry of $\mathbb{Z}_{k}$ parafermions [15]; it would be interesting to develop a physical picture for this symmetry reduction and its interplay with non-abelian statistics (this point will be further discussed in Section 4.5).

More work (mostly numerical analyses) needs to be done for extracting physical information from the quasi-hole wave functions (2.10). As for exact results, using conformal methods, the parent abelian theory presented in this Section is not particularly convenient, because the projection to the parafermion theory is a non-standard mapping between conformal theories with central charges $c=k$ and $c=3 k /(k+2)$, respectively. Therefore, it is useful to consider another parent abelian theory with $c=2 k-1$ : although larger, this theory admits a known mapping to the parafermions, the coset construction (1.2) [15], which yields the complete conformal-theory description of the parafermion quasi-particle excitations.

\section{Maximally symmetric parent abelian theory for the parafermion Hall states}

\subsection{Maximally symmetric $(2 k-1)$-dimensional lattice}

In this Section, we describe the abelian theory with central charge $c=2 k-1$ and affine symmetry $\widehat{u(1)} \oplus \widehat{s u(k)_{1}} \oplus \widehat{s u(k)_{1}}$, which also reproduces the filling fractions (1.1) of the parafermion theory. It is described by a maximally symmetric Hall lattice in the terminology of Ref. 21]. We recall that abelian theories with maximally symmetric lattices reproduce almost all observed filling fractions, including the Jain plateaus [21]; however, the present theory corresponds to different ranges of filling fraction $\nu_{k}$, i.e. the sub-windows [21]:

$$
\Sigma_{p}^{-}=\left\{\nu ; \frac{1}{2 p} \leq \nu<\frac{1}{2 p-1}\right\} \quad \text { for } M=2 p-1 .
$$

As noted in [21], the properties of Hall states in these sub-windows are strikingly

different from the better-known complementary ones with $\nu \in \Sigma_{p}^{+}=\left[\frac{1}{2 p+1}, \frac{1}{2 p}\right)$, that include the Laughlin series $\nu=1 / 3,1 / 5, \ldots$.

We shall briefly recall the notion of maximal symmetry [21]. A chiral Hall lattice is called maximally symmetric if its neutral sub lattice $\left(\Gamma_{0} \perp \underline{Q}\right)$ coincides with the 
(internal symmetry) Witt sub-lattice $\Gamma_{W} \subset \Gamma$ (defined as the sub-lattice generated by all vectors of square length 1 or 2 ) so that $\operatorname{dim} \Gamma_{W}=\operatorname{dim} \Gamma-1$. The Witt sub-lattice is always of the type $A \oplus D \oplus E \oplus \mathbb{Z}$. The maximally symmetric lattices, denoted in [21] by the symbol $\left(L \mid \underline{\omega} \Gamma_{W}\right)$, can be characterized in an appropriate basis by the following Gram matrix:

$$
G=\left[\begin{array}{c|c}
L & \underline{\omega} \\
\hline \underline{\omega}^{T} & C\left(\Gamma_{W}\right)
\end{array}\right]
$$

the charge vector is $Q=(1,0, \ldots, 0)$ in the corresponding dual basis. Here $L \in \mathbb{N}$ is the minimal relative angular momentum that appears as the minimal power of $\left(z_{i}-z_{j}\right)$ in the corresponding wave function; $C\left(\Gamma_{W}\right)$ is the Cartan matrix for the Witt sub-lattice and $\underline{\omega}$ is an admissible weight for $\Gamma_{W}$ restricted by the condition $(\underline{\omega} \mid \underline{\omega})<L$ (see Eq. (5.6) in [21]). Note that the Witt sub-lattice does not contribute to the filling fraction $\nu$ since it is orthogonal to the charge vector $\underline{Q}$. It is easy to find $\nu$ for this lattice using (1.3),

$$
\nu=(\underline{Q} \mid \underline{Q})=Q^{T} \cdot G_{\Gamma}^{-1} \cdot Q=\frac{1}{L-(\underline{\omega} \mid \underline{\omega})} .
$$

The next step is to derive the maximally symmetric Hall lattice which reproduces the filling fraction $\nu_{k}(M)$ in (1.1); we combine Eqs. (3.3) and (1.1) so that:

$$
\frac{1}{L-(\underline{\omega} \mid \underline{\omega})}=\frac{1}{M+\frac{2}{k}}=\frac{1}{M+2-\left(2-\frac{2}{k}\right)} .
$$

We identify $L \equiv M+2$ since $L$ should have the same parity as $M$. In order to find $\underline{\omega}$, we recall that the square of the $i$-th $s u(k)$-fundamental weight is $i(k-i) / k$. Therefore, we can write:

$$
\underline{\omega}=\underline{\Lambda}_{1}^{(\alpha)} \pm \underline{\Lambda}_{1}^{(\beta)}, \quad(\underline{\omega} \mid \underline{\omega})=\left(\underline{\Lambda}_{1}^{(\alpha)} \mid \underline{\Lambda}_{1}^{(\alpha)}\right)+\left(\underline{\Lambda}_{1}^{(\beta)} \mid \underline{\Lambda}_{1}^{(\beta)}\right)=2 \frac{k-1}{k}
$$

where $(\alpha)$ and $(\beta)$ denote two commuting copies of $s u(k)$ with roots $\underline{\alpha}^{i}$ and $\underline{\beta}^{j}$ and fundamental weights $\underline{\Lambda}_{i}^{(\alpha)}$ and $\underline{\Lambda}_{j}^{(\beta)}$ respectively, i.e., $\left(\underline{\Lambda}_{i}^{(\alpha)} \mid \underline{\alpha}^{j}\right)=\left(\underline{\Lambda}_{i}^{(\beta)} \mid \underline{\beta}^{j}\right)=\bar{\delta}_{i}^{j}$, and,

$$
\left(\underline{\alpha}^{i} \mid \underline{\alpha}^{j}\right)=\left(\underline{\beta}^{i} \mid \underline{\beta}^{j}\right)=C^{i j}, \quad\left(\underline{\Lambda}_{i}^{(\alpha)} \mid \underline{\Lambda}_{j}^{(\alpha)}\right)=\left(\underline{\Lambda}_{i}^{(\beta)} \mid \underline{\Lambda}_{j}^{(\beta)}\right)=C_{i j}^{-1}=\frac{i(k-j)}{k}, \quad i \leq j,
$$

where $C^{i j}$ is the $s u(k)$ Cartan matrix (all other inner products vanish). This fixes the Witt sub-lattice to be $\Gamma_{W}=A_{k-1} \oplus A_{k-1}$.

${ }^{*}$ In this case, it is equal to twice the conformal dimension of the electron operator, i.e. an odd integer. 
A convenient basis $\left\{\underline{\mathrm{q}}^{a}\right\}_{a=1}^{2 k-1}$ for $\Gamma$ is given by the electron vector $\mathrm{q}$ (satisfying (1.3) and the spin-statistics relation (1.4)) and the root vectors of $s u(\bar{k}) \oplus s u(k)$ :

$$
\begin{array}{ll}
\underline{\mathrm{q}}^{1}=\underline{\mathrm{q}}, & \\
\underline{\mathrm{q}}^{1+i}=\underline{\alpha}^{i}, & i=1, \ldots, k-1, \\
\underline{\mathrm{q}}^{k+i}=\underline{\beta}^{i}, & i=1, \ldots, k-1 .
\end{array}
$$

One important consequence of the specific structure of the Gram matrix (3.2) is the following decomposition of the electron vector $\underline{q}$ :

$$
\underline{\mathrm{q}}=-\frac{1}{\nu_{k}} \underline{Q}+\underline{\omega}
$$

where $(\underline{Q} \mid \underline{\omega})=0$ and $\left(\underline{\alpha}_{i} \mid \underline{\omega}\right)=\delta_{i 1}=-\left(\underline{\beta}_{i} \mid \underline{\omega}\right)$. Note that the present choice of sign for $\underline{\omega}$ slightly differs from the one in Ref. [16], but it fits the standard notation for coset projections (see Section 4).

The Gram matrix in the basis (3.6) takes the form:

$$
\left[G_{\Gamma}^{a b}\right]=\left[\left(\underline{\mathrm{q}}^{a} \mid \underline{\mathrm{q}}^{b}\right)\right]=\left[\begin{array}{c|c|c}
M+2 & 10 \cdots & -10 \cdots 0 \\
\hline 1 & & \\
0 & C_{k-1} & 0 \\
\vdots & & \\
0 & & C_{k-1} \\
\hline-1 & &
\end{array}\right],
$$

with $C_{k-1}$ the Cartan matrix of the $A_{k-1}$ algebra (recall that the charge vector is $Q=(1,0, \ldots, 0)$ in the dual basis). This completes the derivation of the maximally symmetric abelian lattice theory with $c=2 k-1$ and filling fractions (1.1); its edge excitations are described by representations of the associated chiral algebra $\widehat{u(1)} \oplus \widehat{s u(k)_{1}} \oplus \widehat{s u(k)_{1}}$, which will be discussed in the next Section.

We now describe the decomposition of the lattice (3.6), (3.8) into neutral and charged sub-sectors for the later purpose of projecting out neutral degrees of freedom and obtaining the parafermion conformal theory (Section 4). The strategy is the following: we should first identify the abelian representations with zero charge and the corresponding points in $\Gamma$; next, we single out a sub-lattice $\Gamma$ which is the direct sum of a one-dimensional charge sub-lattice and its orthogonal complement; finally, we only project the neutral points which are orthogonal to the electron vector (3.7) (and also orthogonal to $\underline{\omega}$ ). The last condition implies that the conformal dimension of the electron, i.e. the spin-statistics relation, is preserved by the projection:

$$
\Delta_{\mathrm{el}}=\frac{1}{2}(\underline{\mathrm{q}} \mid \underline{\mathrm{q}})=\frac{1}{2}\left(\frac{1}{\nu_{k}}+(\underline{\omega} \mid \underline{\omega})\right)=\frac{M+2}{2} ;
$$


(note that $\underline{\omega}$ yields a crucial contribution according to Eq. (3.7)).

The unitary representations of the chiral algebra $\mathcal{A}(\Gamma)$ (describing the edge excitations) are labelled by the points of the dual lattice $\Gamma^{*}$ : this is manifestly not decomposable into orthogonal sub-lattices of charge and neutral excitations. Nevertheless, this decomposition (an instance of the isospin-charge separation) can be achieved at the expenses of enlarging the dual lattice (of excitations) and of introducing a selection rule (the $\mathbb{Z}_{k}$ parity rule). We introduce the decomposable sub-lattice $L \subset \Gamma$ of index $k$ spanned by the vectors:

$$
\left\{\underline{\mathrm{e}}^{1}, \underline{\alpha}_{i}, \underline{\beta}_{j}\right\}, \quad \underline{\mathrm{e}}^{1}=k(\underline{\mathrm{q}}-\underline{\omega})=(k M+2) \underline{Q} .
$$

It splits into 3 mutually orthogonal sub-lattices:

$$
L=(k M+2) \mathbb{Z} \underline{Q} \oplus A_{k-1} \oplus A_{k-1} .
$$

We have

$$
\begin{array}{ll}
L \subset \Gamma \subset \Gamma^{*} \subset L^{*}, \quad & \Gamma=\{\underline{\gamma}=\underline{\lambda}+n \underline{\mathrm{q}} ; \underline{\lambda} \in L, 0 \leq n \leq k-1\}, \\
& L^{*} / \Gamma^{*} \simeq \Gamma / L \simeq \mathbb{Z}_{k} ;
\end{array}
$$

indeed, the determinants of the Gram matrices of $L$ and $\Gamma$ (which give the number of sectors of the corresponding rational conformal theory) are:

$$
|L|=(k M+2)^{2}|\underline{Q}|^{2}\left|C_{k-1}\right|^{2}=(k M+2) k^{3}=k^{2}|\Gamma| .
$$

The isospin-charge separation of excitations is achieved in the decomposable dual lattice $L^{*}$, whose physical points (corresponding to the points of $\Gamma^{*}$ ) obey a selection rule $\bmod k$. This is the desired factorization of excitations in the abelian theory which will allow the projection of neutral degrees of freedom.

\subsection{Unitary irreducible representations of the chiral algebra $\mathcal{A}(\Gamma)$. The $\mathbb{Z}_{k}$ parity rule}

The irreducible unitary representations of the chiral algebra $\mathcal{A}(L)$ can be expressed as $\mathbb{Z}_{k}$-invariant products of fundamental representations of $\widehat{s u(k)_{1}} \oplus \widehat{s u(k)_{1}}$ times chiral vertex operators carrying charge $(n / k) \underline{Q}$, with $n \in \mathbb{Z} / k(k M+2) \mathbb{Z}$; these representations are labelled by the elements of the abelian group $L^{*} / L$. We shall choose a vector $\underline{\lambda} \in L^{*}$ in each coset in $L^{*} / L$ of the form:

$$
\underline{\lambda}=m \underline{\mathrm{e}}_{1}^{*}+\underline{\Lambda}_{\mu}^{(\alpha)}-\underline{\Lambda}_{\rho}^{(\beta)}, \quad \mu, \rho=0,1, \ldots, k-1, \quad 2|m| \leq k(k M+2) .
$$

Here $\underline{\Lambda}_{\mu}^{(\alpha)}\left(\underline{\Lambda}_{\rho}^{(\beta)}\right)$ are the fundamental weights (including 0) of the first (respectively, the second) $s u(k)$ factor. The conformal dimension of the representation $\underline{\lambda}$ is:

$$
\Delta(\underline{\lambda})=\frac{1}{2}|\underline{\lambda}|^{2}=\frac{m^{2}}{2 k(k M+2)}+\frac{\mu(k-\mu)+\rho(k-\rho)}{2 k} .
$$


The charges $\gamma^{*} \in \Gamma^{*} / \Gamma$ representing the excitations of the chiral algebra $\mathcal{A}(\Gamma)$ could be viewed as points in $L^{*} / L$ since we have $\Gamma^{*} / \Gamma \subset L^{*} / L$ by Eq.(3.12); this is convenient because of the spin-charge decomposition of $L$ and $L^{*}$. However, we need a criterion to select the points of the original dual lattice $\Gamma^{*}$ among those of the bigger lattice $L^{*}$ : since $\underline{q}$ is the only vector in the basis of $\Gamma$ which does not belong to $L$, we find the following natural condition.

Proposition 3.1 A vector $\underline{\lambda}$ of $L^{*}$ belongs to the sub-lattice $\Gamma^{*}$ if and only if $(\underline{\lambda} \mid \underline{q}) \in$ $\mathbb{Z}$. For $\underline{\lambda}$ given by (3.14), this is equivalent to the relation:

$$
m+k\left(\underline{\omega} \mid \underline{\Lambda}_{\mu}^{(\alpha)}-\underline{\Lambda}_{\rho}^{(\beta)}\right) \in k \mathbb{Z} \quad \Longleftrightarrow \quad \mu+\rho=m \bmod k .
$$

Proof. Since $\underline{q} \in \Gamma$ and $\underline{\lambda} \in \Gamma^{*}$ the inner product $(\underline{\lambda} \mid \underline{q})$ is integer. For $\underline{\lambda} \in L^{*}$ the converse is also true in view of (3.7) and (3.11). Eq. (3.16) is then a consequence of (1.3) (3.7) and (3.11) and of the definition of fundamental weights as a dual basis for $\left\{\underline{\alpha}_{i}\right\}$ and $\left\{\underline{\beta}_{i}\right\}$. As a result, the inner products $\left(\underline{\Lambda}_{i} \mid \underline{\Lambda}_{j}\right)$ (for $\underline{\Lambda}$ belonging to the weight space of the same $s u(k)$ factor, $\alpha$ or $\beta$ ) are expressed in terms of the inverse $A_{k-1}$ Cartan matrix.

Equation (3.16) is the explicit form of the $\mathbb{Z}_{k}$ parity rule which selects the points in $L^{*}$ that also belong to the sub-lattice $\Gamma^{*}$ of physical excitations of the Hall state.

According to Eq. (3.12) the charges labelling the irreducible representations of $\mathcal{A}(\Gamma)$ can be written in the basis of $L^{*}$ as follows, taking into account (3.14) and (3.16):

$$
\begin{aligned}
\underline{\gamma}^{*}=\underline{\lambda}+n \underline{\mathrm{q}} & =m \underline{\mathrm{e}}_{1}^{*}+\underline{\Lambda}_{\rho+m}^{(\alpha)}-\underline{\Lambda}_{k-\rho}^{(\beta)}+n\left(\frac{k}{\nu_{k}} \underline{\mathrm{e}}_{1}^{*}+\underline{\Lambda}_{1}^{(\alpha)}-\underline{\Lambda}_{1}^{(\beta)}\right) \\
& =(m+n(k M+2)) \underline{\mathrm{e}}_{1}^{*}+\underline{\Lambda}_{\rho+n+m}^{(\alpha)}-\underline{\Lambda}_{n-\rho}^{(\beta)},
\end{aligned}
$$

where we have used the fact that the sum of $s u(k)$ fundamental weights $\underline{\Lambda}_{\mu}+\underline{\Lambda}_{\rho}$ belongs to the sector of $\underline{\Lambda}_{\mu+\rho \bmod k}$. Therefore, we can label the irreducible representations of $\mathcal{A}(\Gamma)$ by a pair $(m, \rho)$ corresponding to Eq. (3.17), where $m$ measures the minimal charge of each irreducible representation so that $2|m| \leq(k M+2)$, while $\rho \bmod k$ characterizes the neutral part.

Using the representation of each vector $\underline{\gamma} \in \Gamma$ as a sum of an $\underline{\lambda} \in L$ and a gluing vector [21] $n \underline{\mathrm{q}} \in \Gamma$ (see (3.12)), we can write the representation space $\mathcal{H}_{\underline{\lambda}}^{\Gamma}$ of $\mathcal{A}(\Gamma)$ as direct sums of representation spaces of $\mathcal{A}(L)$,

$$
\mathcal{H}_{\underline{\lambda}}^{\Gamma}=\bigoplus_{n=0}^{k-1} \mathcal{H}_{\underline{\lambda}+n \underline{q}}^{L},
$$

where $\underline{\lambda} \in L^{*} / L$ is supposed to satisfy the parity rule (3.16), so that $\underline{\lambda} \in \Gamma^{*} / \Gamma$. This form of $\mathcal{H}_{\underline{\lambda}}^{\Gamma}$ allows to write the corresponding characters $\chi_{\underline{\underline{\lambda}}}^{\Gamma}(\tau, \zeta)=\operatorname{tr}_{\mathcal{H}_{\underline{\lambda}}^{\Gamma}} q^{L_{0}-c / 24} e^{2 \pi i\left(\underline{Q} \mid \underline{J_{0}}\right)}$ 
as sums of characters of $\mathcal{A}(L)$ modules:

$$
\chi_{\underline{\lambda}}^{\Gamma}(\tau, \zeta)=\sum_{n=0}^{k-1} \chi_{\underline{\lambda}+n \underline{q}}^{L}(\tau, k \zeta)
$$

where the factor $k$ in the argument $k \zeta$ comes from the normalization of the charge vector in the basis of $L^{*}$. This expression is convenient since the character $\chi_{\underline{\lambda}+n \underline{\mathrm{q}}}^{L}(\tau, k \zeta)$ of $\mathcal{A}(L)$ is simply the product of a one-dimensional lattice character [16] (corresponding to the charged sub-lattice):

$$
K_{l}(\tau, \zeta ; p)=\frac{1}{\eta(\tau)} \sum_{n \in \mathbb{Z}} q^{\frac{p}{2}\left(n+\frac{l}{p}\right)^{2}} \mathrm{e}^{2 \pi i \zeta\left(n+\frac{l}{p}\right)},
$$

and two copies of the (restricted, i.e., neutral) $\widehat{s u(k)_{1}}$ characters $\chi_{\rho}(\tau)$ [27],

$$
\chi_{\rho}\left(\tau, A_{k-1}\right)=\frac{1}{(\eta(\tau))^{k-1}} \sum_{\underline{\gamma} \in A_{k-1}} q^{\frac{1}{2}\left|\underline{\Lambda}_{\rho}+\underline{\gamma}\right|^{2}}, \quad q=\mathrm{e}^{2 \pi i \tau}, \quad \rho=0,1, \ldots, k-1 .
$$

( $\eta$ is the Dedekind function and $A_{k-1}$ is the root lattice). Therefore, we can write the characters $\chi_{\underline{\lambda}}(\tau, \zeta)$ of the representation $\underline{\lambda}$ (3.14) as sums of factorized $L$-characters,

$$
\chi_{m, \rho}^{\Gamma}(\tau, \zeta)=\sum_{n=0}^{k-1} K_{m+n(k M+2)}(\tau, k \zeta ; k(k M+2)) \chi_{\rho+m+n}^{(\alpha)}(\tau) \chi_{n-\rho}^{(\beta)}(\tau),
$$

with $m \bmod (k M+2)$ and $\rho \bmod k$. Note that, originally $m$ was an index defined $\bmod k(k M+2)$; however, Eq. (3.22) shows that the characters are $\mathbb{Z}_{k}$ invariant, $\chi_{m+(k M+2), \rho}^{\Gamma}(\tau, \zeta)=\chi_{m, \rho}^{\Gamma}(\tau, \zeta)$, so that there are just $(k M+2)$ independent values for $m$. The resulting set of $k(k M+2)$ functions is covariant under (weak) modular transformations generated by $T^{2}:(\tau, \zeta) \rightarrow(\tau+2, \zeta)$ and $S:(\tau, \zeta) \rightarrow(-1 / \tau, \zeta / \tau)$, which are the modular properties suitable for quantum Hall systems [22].

\section{The $\mathbb{Z}_{k}$-parafermion coset and its representa- tions}

In this Section, we describe the coset construction (1.2); this can be done in each of the $k$ sectors (3.18) of the abelian theory according to the $\mathbb{Z}_{k}$ parity rule.

\section{1 $\mathbb{Z}_{k}$ selection rule for triples of $\mathrm{su}(\mathrm{k})$ weights. Conformal dimensions of coset representations}

The $\mathrm{PF}_{k}$ coset module (1.2) is labelled, in principle, by a triple $\left(\underline{\Lambda}_{\alpha}, \underline{\Lambda}_{\beta} ; \underline{\Lambda}\right)$; the pair $\left(\underline{\Lambda}_{\alpha}, \underline{\Lambda}_{\beta}\right)$ of fundamental $s u(k)$ weights and the level 2 weight $\underline{\Lambda}$ fix an irreducible 
unitary representation of the numerator and of the denominator current algebra,

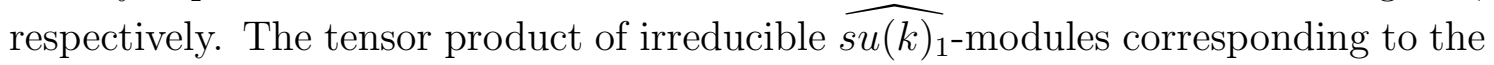
numerator in the right hand side of Eq. (1.2) splits into a direct sum of $\mathrm{PF}_{k}$ and $\widehat{s u(k)_{2}}$-modules:

$$
\mathcal{H}_{\underline{\Lambda}_{\alpha}}^{(1)} \otimes \mathcal{H}_{\underline{\Lambda}_{\beta}}^{(1)}=\bigoplus_{\underline{\Lambda}} \mathcal{H}\left(\underline{\Lambda}_{\alpha}, \underline{\Lambda}_{\beta} ; \underline{\Lambda}\right) \otimes \mathcal{H}_{\underline{\Lambda}}^{(2)} \quad\left(\alpha, \beta=0, \ldots, k-1, \underline{\Lambda}_{0}=0\right) .
$$

Not all triples $\left(\underline{\Lambda}_{\alpha}, \underline{\Lambda}_{\beta} ; \underline{\Lambda}\right)$ are admissible (i.e., correspond to non-empty coset modules) and different admissible triples may refer to equivalent representations. The following statement is a specialization of results on field identification (based on the use of simple currents) obtained in Ref. 28.

Proposition 4.1 Admissible triples are characterized by the conservation of the $\mathbb{Z}_{k}$ charge given by the k-ality:

$$
[\underline{\Lambda}]=\sum_{i=1}^{k-1} i \lambda_{i} \quad \text { for } \quad \underline{\Lambda}=\sum_{i=1}^{k-1} \lambda_{i} \underline{\Lambda}_{i}
$$

more precisely, the triple $\left(\underline{\Lambda}_{\alpha}, \underline{\Lambda}_{\beta} ; \underline{\Lambda}\right)$ is admissible if and only if:

$$
\left[\underline{\Lambda}_{\alpha}\right]+\left[\underline{\Lambda}_{\beta}\right]=[\underline{\Lambda}] \bmod k, \quad \text { i.e., } \quad \alpha+\beta=[\underline{\Lambda}] \bmod k \text {. }
$$

There are thus $k\left(\begin{array}{c}k+1 \\ 2\end{array}\right)$ admissible triples of the form $\left(\underline{\Lambda}_{\alpha}, \underline{\Lambda}_{\beta} ; \underline{\Lambda}_{\alpha+\kappa}+\underline{\Lambda}_{\beta-\kappa}\right)$ where all indices are taken $\bmod k$. They split into $\left(\begin{array}{c}k+1 \\ 2\end{array}\right)$ families of equivalent triples of the form:

$$
\left(\underline{\Lambda}_{\alpha+\sigma}, \underline{\Lambda}_{\beta+\sigma} ; \underline{\Lambda}_{\alpha+\kappa+\sigma}+\underline{\Lambda}_{\beta-\kappa+\sigma}\right), \quad \sigma=0, \ldots, k-1 .
$$

As a result, the number $N\left(\mathrm{PF}_{k}\right)$ of parafermion coset sectors coincides with the number of unitary irreducible representation $N\left(\widehat{s u(k)_{2}}\right)$ of the level 2 current algebra: $N\left(\mathrm{PF}_{k}\right)=N\left(\widehat{s u(k)_{2}}\right)=k(k+1) / 2$.

We can define a representative for each family in Eq.(4.4) by choosing a value for $\sigma$ : we set $\beta+\sigma=0 \bmod k$, thus normalizing the second fundamental weight to zero. Therefore, the equivalent classes of triples can be labelled by the level-two weight, say $\underline{\Lambda}_{\mu}+\underline{\Lambda}_{\rho}, \mu \leq \rho$; we have:

$$
\left(\underline{\Lambda}_{\mu+\rho \bmod k}, 0 ; \underline{\Lambda}_{\mu}+\underline{\Lambda}_{\rho}\right) \Longleftrightarrow \underline{\Lambda}_{\mu}+\underline{\Lambda}_{\rho} \quad(\mu \leq \rho) .
$$

Ultimately, these labels characterize the parafermion representations. We end up with the following characterization of $\mathrm{PF}_{k}$ coset modules which appears to be new. 
Proposition 4.2 The parafermion coset modules are in one to one correspondence with sums $\left(\underline{\Lambda}_{\mu}+\underline{\Lambda}_{\rho}\right)$ of su( $\left.k\right)$ fundamental weights $(0 \leq \mu \leq \rho \leq k-1)$. Their conformal weights are given by:

$$
\begin{aligned}
\Delta_{\mu \rho}^{(k)} & =\frac{1}{2}\left|\underline{\Lambda}_{\mu}+\underline{\Lambda}_{\rho}\right|^{2}-\Delta_{2}\left(\underline{\Lambda}_{\mu}+\underline{\Lambda}_{\rho}\right) \\
& =\frac{\mu(k-\rho)}{k}+\frac{(\rho-\mu)(k+\mu-\rho)}{2 k(k+2)} \text { for } \quad(0 \leq \mu \leq \rho<k),
\end{aligned}
$$

where $\Delta_{2}(\underline{\Lambda})$ is the dimension of the representation of the level 2 weight $\underline{\Lambda}$ of $\widehat{s u(k)_{2}}$. The decomposition of a product of level-1 characters corresponding to the tensor product expansion (4.1) for any triple (4.5) has the form:

$$
\chi^{(1)}\left(\underline{\Lambda}_{\underline{\alpha}-\underline{\beta}}\right) \chi^{(1)}\left(\underline{\Lambda}_{0}\right)=\sum_{\gamma} \operatorname{Ch}\left(\underline{\Lambda}_{\alpha-\beta+\gamma}+\underline{\Lambda}_{k-\gamma}\right) \chi^{(2)}\left(\underline{\Lambda}_{\alpha-\beta+\gamma}+\underline{\Lambda}_{k-\gamma}\right),
$$

where $\mathrm{Ch}(\underline{\Lambda})$ is the coset character and $\gamma$ takes all values corresponding to distinct sums of pairs of weights (they are no more than $(k / 2)+1)$.

Proof. Eq. (4.6) can be verified by using the identity $\Delta_{1}\left(\underline{\Lambda}_{\mu}\right)+\Delta_{1}\left(\underline{\Lambda}_{k-\rho}\right)-$ $\Delta_{2}\left(\underline{\Lambda}_{\mu+k-\rho}\right)=\Delta_{\mu \rho}^{(k)}$ and observing that the triple $\left(\underline{\Lambda}_{\mu}, \underline{\Lambda}_{k-\rho} ; \underline{\Lambda}_{\mu+k-\rho}\right)$ is equivalent to $\left(\underline{\Lambda}_{\mu+\rho}, 0 ; \underline{\Lambda}_{\mu}+\underline{\Lambda}_{\rho}\right)$. The triples appearing in Eq. (4.7) (as arguments of the pair of $\chi^{(1)}$ and $\chi^{(2)}$ ) are, clearly, admissible. It is straightforward to verify that the difference of conformal weights of the two sides is an integer. The number of terms in the expansion (4.7) is independent of $\alpha$ and $\beta$. For the vacuum representation we have, for example:

$$
\chi^{(1)}\left(\underline{\Lambda}_{0}\right) \chi^{(1)}\left(\underline{\Lambda}_{0}\right)=\operatorname{Ch}\left(2 \underline{\Lambda}_{0}\right) \chi^{(2)}\left(2 \underline{\Lambda}_{0}\right)+\sum_{\gamma=1}^{\operatorname{IP}\left(\frac{k}{2}\right)} \operatorname{Ch}\left(\underline{\Lambda}_{\gamma}+\underline{\Lambda}_{k-\gamma}\right) \chi^{(2)}\left(\underline{\Lambda}_{\gamma}+\underline{\Lambda}_{k-\gamma}\right),
$$

where $\Delta_{\gamma, k-\gamma}^{(k)}+\Delta_{2}\left(\underline{\Lambda}_{\gamma}+\underline{\Lambda}_{k-\gamma}\right)=\gamma$ for $\gamma \leq k-\gamma$, and $\operatorname{IP}(x)$ stands for the integer part of the real number $x$.

\subsection{Parafermion Hall states. The parafermion $\mathbb{Z}_{k}$ charge}

The chiral algebra $\mathcal{A}_{k}$ of the $\mathbb{Z}_{k}$-parafermion Hall states, with filling fraction (1.1), is determined from:

$$
\mathcal{A}_{k} \otimes \mathcal{A}\left(\widehat{s u(k)_{2}}\right)=\mathcal{A}(\Gamma) .
$$

This is obtained by coset projection from the lattice theory of Section 3. In particular, the lattice characters (3.22) are projected into:

$$
\chi_{m \rho}(\tau, \zeta)=\sum_{s \bmod k} K_{m+s(k M+2)}(\tau, k \zeta ; k(k M+2)) \operatorname{Ch}\left(\tau, \underline{\Lambda}_{s+m+\rho}+\underline{\Lambda}_{s-\rho}\right) .
$$


The coset projection only preserves a single $\mathbb{Z}_{k}$ symmetry of the original product $\mathbb{Z}_{k} \times \mathbb{Z}_{k}$ of the centres of the two $s u(k)$ groups; this is:

$$
\left[\underline{\Lambda}_{\mu}+\underline{\Lambda}_{\rho}\right]=\mu+\rho \quad \bmod k
$$

which defines the $\mathbb{Z}_{k}$ charge of parafermions $p=\mu+\rho$.

Moreover, the $\mathbb{Z}_{k}$ parity rule of the parafermion Hall states is inherited from the corresponding selection rule of the parent abelian state, given by Eq.(3.14): this requires that the physical Hall excitations possess parafermion "charge" (4.10) equal $(\bmod k)$ to the number of "fractional units" of electric charge $l \in \mathbb{Z}_{k M+2}$ in Eq.(3.14) (see also Eq.(3.16)):

$$
p=\mu+\rho=m \bmod k .
$$

The coset representations $2 \underline{\Lambda}_{\rho}$, corresponding to $\mu=\rho$, are the "parafermion currents" of Fateev and Zamolodchikov [9]. They are "simple currents" [28] obeying $\mathbb{Z}_{k}$ fusion rules:

$$
2 \underline{\Lambda}_{\mu} \times 2 \underline{\Lambda}_{\rho} \sim 2 \underline{\Lambda}_{(\mu+\rho) \bmod k} .
$$

The (non-local) parafermion currents give rise to an "anyonic" chiral algebra, say, $\mathcal{P} \mathcal{F}_{k}$ whose bosonic (integer dimension fields') sub-algebra can be identified with the coset chiral algebra $\mathrm{PF}_{k}(1.2)$. The parafermion algebra $\mathcal{P} \mathcal{F}_{k}$ admits $k$ unitary irreducible representations, labelled by an integer $\sigma \bmod k$, with conformal weights,

$$
\Delta_{\sigma}=\frac{\sigma(k-\sigma)}{2 k(k+2)}, \quad \sigma=0,1, \ldots, k-1
$$

Each of these splits into $(k-\sigma)$ unitary irreducible representation of the bosonic subalgebra $\mathrm{PF}_{k}$ whose conformal weights exceed (4.13) by an integer multiple of $1 / k$. Comparing (4.13) with (4.6) we see that $\sigma$ can be identified with $(\rho-\mu)$. For each $\sigma$ in the range (4.13) there are exactly $(k-\sigma)$ pairs $(\mu, \rho)$ satisfying $0 \leq \mu \leq \rho \leq k$, $\sigma=\rho-\mu$; they generate all different conformal weights (4.6). Following Ref. [29], the characters of the resulting coset modules are given by $\operatorname{Ch}\left(\tau, \underline{\Lambda}_{\mu}+\underline{\Lambda}_{\rho}\right)=\mathrm{Ch}_{\sigma l}(\tau)$, where:

$$
\mathrm{Ch}_{\sigma l}(\tau)=q^{\Delta_{\sigma}-\frac{c_{k}-1}{24}} \sum_{\underline{n} \in \mathbb{N}_{l}} \frac{q^{\underline{n} \cdot C^{-1} \cdot\left(\underline{n}-\underline{\Lambda}_{\sigma}\right)}}{(q)_{n_{1}} \cdots(q)_{n_{k-1}}}, \quad l \geq \sigma .
$$

In this equation, $\Delta_{\sigma}$ is the $\mathrm{PF}_{k}$ weight $(4.13),\left(c_{k}-1\right)$ is the parafermion central charge (cf. (1.2)), $(q)_{n}=\prod_{j=1}^{n}\left(1-q^{j}\right), \mathbb{N}_{l}=\left\{\underline{n}=\left(n_{1}, \ldots, n_{k-1}\right) ; n_{i} \in \mathbb{Z}_{+}\right.$; $\left.n_{1}+2 n_{2}+\cdots+(k-1) n_{k-1}=l \bmod k\right\}$, and $C^{-1}$ is the inverse of the $s u(k)$ Cartan matrix.

The expression (4.14) corresponds to a $\mathrm{PF}_{k}$ irreducible component of the representation $\sigma$ of the non-local parafermion algebra $\mathcal{P \mathcal { F }}_{k}$. For fixed $\sigma$, the values of $l$ yielding inequivalent $\mathrm{PF}_{k}$ modules are:

$$
l=\sigma, \sigma+1, \ldots, k-1 \quad(\text { for } \sigma=0,1, \ldots, k-1) .
$$


The pair $(\sigma, l)$ is related to $(\mu, \rho)$ of (4.6) by:

$$
\sigma=\rho-\mu, l=\rho \quad \text { i.e. } \quad \mu=l-\sigma, \rho=l \quad \Rightarrow \quad 2 l-\sigma \equiv m \bmod k .
$$

The topological order $N_{k}$ of the $\mathbb{Z}_{k}$-parafermion Hall state is equal [22] to the number of independent characters (4.9) of the corresponding conformal theory; it is given by the product of the range of $m$, i.e. $(k M+2)$, multiplied by the number $\left(\begin{array}{c}k+1 \\ 2\end{array}\right)$ of $\mathbb{Z}_{k}$-parafermion sectors and divided by $k$, due to the parity rule (4.11):

$$
N_{k}=\frac{1}{k}(k M+2)\left(\begin{array}{c}
k+1 \\
2
\end{array}\right)=\frac{k+1}{2}(k M+2) \text {. }
$$

We thus recover the value obtained in Ref. [8] by other means.

\subsection{Quasi-particle excitations of parafermion Hall states}

The charge and conformal dimensions of all particles in the theory can be deduced from their characters - note that the characters (3.22) and (4.9) are represented as sums of product of "charged" and "neutral" characters that always satisfy the $\mathbb{Z}_{k}$ parity rule. In the coset, the charge and total conformal dimension of each term $K_{m+s(k M+2)}(\tau, k \zeta ; k(k M+2)) \operatorname{Ch}\left(\tau, \underline{\Lambda}_{s+m+\rho}+\underline{\Lambda}_{s-\rho}\right)$ in Eq. (4.9) are given by:

$$
\begin{aligned}
& Q=k \frac{m+s(k M+2)}{k(k M+2)}=\frac{m+s(k M+2)}{k M+2}, \\
& \Delta_{\text {tot }}=\frac{(m+s(k M+2))^{2}}{2 k(k M+2)}+\Delta^{(k)}\left(\underline{\Lambda}_{l+\sigma+s}+\underline{\Lambda}_{s-\sigma}\right) .
\end{aligned}
$$

The physical hole operator (whose charge conjugate is the electron operator) is identified with the tensor product:

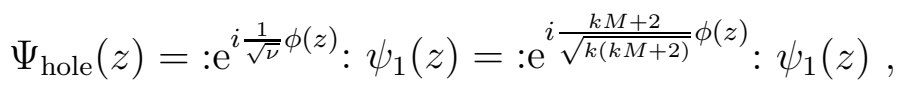

satisfying the parity rule (4.11) $\left(\psi_{1}\right.$ being the $l=1$ parafermion field in Eq.(1.13)). The sector corresponding to one hole is given by the $s=1$ term in the character,

$$
\chi_{00}(\tau, \zeta)=\sum_{s=0}^{k-1} K_{s(k M+2)}(\tau, k \zeta ; k(k M+2)) \operatorname{Ch}\left(\underline{\Lambda}_{s}+\underline{\Lambda}_{s}\right),
$$

so that its charge and conformal dimension are:

$$
Q_{\text {hole }}=1, \quad \Delta_{\text {hole }}=\frac{1}{2 \nu_{k}}+\frac{k-1}{k}=\frac{M+2}{2} .
$$

Note that the conformal dimension of the physical electron (or hole) is independent of $k$. 
Similarly the quasi-hole operator is identified with:

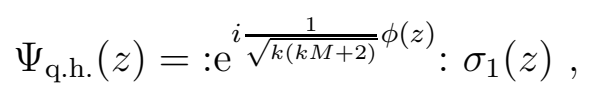

where $\sigma_{1}$ is the spin field with the label $\left(\underline{\Lambda}_{0}+\underline{\Lambda}_{1}\right)$ corresponding to the lowest charge and dimension among the irreducible representations of the coset (see Eq.(1.16)). It is also worth noting that it generates all other irreducible representations by fusion with itself. It is characterized by the $s=0$ term in

$$
\chi_{10}(\tau, \zeta)=\sum_{s=0}^{k-1} K_{1+s(k M+2)}(t, k \zeta ; k(k M+2)) \operatorname{Ch}\left(\tau, \underline{\Lambda}_{s}+\underline{\Lambda}_{s+1}\right),
$$

so that the charge and total conformal dimensions are:

$$
Q_{\text {q.h. }}=\frac{1}{k M+2}, \quad \Delta_{\text {q.h. }}=\frac{1}{2 k(k M+2)}+\frac{k-1}{2 k(k+2)} \stackrel{(M=1)}{=} \frac{1}{2(k+2)} .
$$

\subsection{Coset description of parafermion fusion rules}

All fusions rules can be obtained from those of the basic fields $\underline{\Lambda}_{0}+\underline{\Lambda}_{\mu}, 0 \leq \mu \leq k-1$ (see Eq.(4.5)), taking into account the symmetry of the fusion coefficients under the charge conjugation and the action of the simple currents:

$$
\left(\underline{\Lambda}_{0}+\underline{\Lambda}_{\mu}\right) \cdot\left(\underline{\Lambda}_{0}+\underline{\Lambda}_{\rho}\right)=\left\{\begin{array}{cl}
\bigoplus_{s=0}^{\min (\mu, \rho)}\left(\underline{\Lambda}_{s}+\underline{\Lambda}_{\mu+\rho-s}\right) & \text { if } \quad \min (\mu, \rho) \leq \operatorname{IP}(k / 2), \\
\bigoplus_{s=k-\max (\mu, \rho)}^{k}\left(\underline{\Lambda}_{s}+\underline{\Lambda}_{\mu+\rho-s}\right) & \text { otherwise } .
\end{array}\right.
$$

Note that the second case $\min (\mu, \rho)>\operatorname{IP}(k / 2)$ in Eq. (4.24) is obtained from the first one by using the charge conjugation symmetry $C$ of the fusion coefficients $N_{C(\underline{\underline{\lambda}}), C(\underline{\mu})}^{C(\underline{\rho})}=N_{\underline{\underline{\lambda}}, \underline{\mu}}^{\underline{\rho}}$ where $\underline{\lambda}, \underline{\mu}, \underline{\rho}$ are labels in the form of Eq. 4.5$)$ and $C\left(\underline{\Lambda}_{\mu}+\underline{\Lambda}_{\rho}\right)=$ $\left(\underline{\Lambda}_{k-\mu}+\underline{\Lambda}_{k-\rho}\right)$.

The other fusions could be obtained by the action of the simple currents,

$$
N_{J(\underline{\lambda}), J^{\prime}(\underline{\mu})}^{\left.J . J^{\prime}\right)}=N_{\underline{\underline{\lambda}}, \underline{\mu}}^{\rho}
$$

where $J$ and $J^{\prime}$ are arbitrary powers (up to $k$ ) of the generator $I$ of the simple currents orbit:

$$
I\left(\underline{\Lambda}_{\mu}+\underline{\Lambda}_{\rho}\right)=\left(\underline{\Lambda}_{\mu+1}+\underline{\Lambda}_{\rho+1}\right)
$$


For example, applying $I \times I$ to both sides of Eq. (4.24) (for $\mu \leq \operatorname{IP}(k / 2)$ ) we get:

$$
\begin{aligned}
\left(\underline{\Lambda}_{1}+\underline{\Lambda}_{\mu+1}\right) \cdot\left(\underline{\Lambda}_{1}+\underline{\Lambda}_{\rho+1}\right) & =I\left(\underline{\Lambda}_{0}+\underline{\Lambda}_{\mu}\right) \cdot I\left(\underline{\Lambda}_{0}+\underline{\Lambda}_{\rho}\right)=\bigoplus_{s=0}^{\min (\mu, \rho)} I^{2}\left(\underline{\Lambda}_{s}+\underline{\Lambda}_{\mu+\rho-s}\right) \\
& =\bigoplus_{s=0}^{\min (\mu, \rho)}\left(\underline{\Lambda}_{s+2}+\underline{\Lambda}_{\mu+\rho-s+2}\right) .
\end{aligned}
$$

A complete list of fusion rules for the coset irreducible representations will be given in Section 5 for the $k=3$ case.

\section{5 $\mathcal{W}_{k}$ symmetry of the parafermions: the spectrum of $\mathcal{W}_{k}$ charges in the coset $\widehat{s u(k)_{1}} \oplus \widehat{s u(k)_{1}} / \widehat{s u(k)_{2}}$.}

A characteristic property of the parafermion coset (1.2) is its preservation of the chiral algebra $\mathcal{W}_{k}$ generated by quasi-primary fields of dimensions $2, \ldots, k$ corresponding to the $s u(k)$ polynomial Casimir operators (the quadratic one being the Virasoro stress tensor); instead, the dimension-one currents of the denominator $\widehat{s u(k)_{2}}$ are all gauged away. Altogether, the symmetry $\widehat{s u(k)_{1}} \oplus \widehat{s u(k)_{1}}$ of the parent abelian theory is reduced to the Casimir algebra $\mathcal{W}_{k}$. This result follows from the fact that the conformal fields corresponding to all the Casimir operators commute with the action of the simple currents. Comparison of Eq. (1.2) with the formula for the Virasoro central charge of the $\mathcal{W}_{k}$-symmetric minimal conformal theories [15],

$$
c_{p}\left(\mathcal{W}_{k}\right)=(k-1)\left(1-\frac{k(k+1)}{p(p+1)}\right), \quad p=k+1, k+2, \ldots,
$$

indicates that the $\mathbb{Z}_{k}$-parafermion theories correspond to the lowest models in each series, i.e. $p=k+1$.

The occurrence of the $\mathcal{W}_{k}$ symmetry in the parafermion Hall states is rather intriguing because it also characterizes the better-known Jain Hall states [1]: according to the proposal of Ref. [14], the Jain states are described by conformal theories corresponding to the highest models in the series (4.26), at the limiting value $p=\infty$, which have been called " $W_{1+\infty}$ minimal models". Both realizations of the $\mathcal{W}_{k}$ symmetry come from projections of abelian theories, which are symmetric under the larger algebra $W_{1+\infty}\left(\widehat{u(1)} \oplus \mathcal{W}_{k} \subset W_{1+\infty}\right)$. The latter algebra describes the deformations of planar incompressible fluids under area-preserving diffeomorphisms of the plane; thus, it accounts for the dynamical symmetry of "simple" incompressible Hall fluids 13 .

The projection leading to the $W_{1+\infty}$ minimal models is rather mild, it reduces the multiplicities of the excitations but does not change the central charge [14]: 
the physical picture associated to the basic incompressible Hall fluids, such as the Laughlin fluids, is preserved [13. On the other hand, the projection leading to the parafermion theories is much stronger and the physics of the resulting Hall fluids is rather different from that of the Jain states (and of generic abelian states), as discussed in the previous Sections.

In spite of the differences, the appearance of the $\mathcal{W}_{k}$ symmetry in both cases suggests the possibility of a simple physical mechanism underlying both stable Hall states. For this reason, it is interesting to briefly describe the characterization of the $\mathbb{Z}_{k}$-parafermion sectors as irreducible representations of the $\mathcal{W}_{k}$ algebra; one needs the eigenvalues of the zero modes of all $(k-1) \mathcal{W}_{k}$ "currents" (the first of them, the stress tensor, giving the conformal dimension) to label the lowest-weight states. Of course, in Section 4.1 we introduced the simpler labelling by two quantum numbers: $\mu$ and $\rho(\mu \leq \rho)$ or $\sigma$ and $l$. (The second choice is more convenient as it gives the $\mathbb{Z}_{k}$ charge and the label $l-\sigma$ of the simple current (of weight $2 \underline{\Lambda}_{l-\sigma}$ ) acting on the lowest weight state $\underline{\Lambda}_{0}+\underline{\Lambda}_{\sigma}$.)

The properties of the $\mathcal{W}_{k}$ minimal models are extensively discussed in the review by Bouwknegt and Schoutens [15]; we are going to use their formula (6.50) for the spectrum of the $\mathcal{W}_{k}$ operators derived by the quantum Miura transformation [15]:

$$
\widetilde{\mathcal{W}}_{a}(\underline{\Lambda})=(-1)^{a-1} \sum_{1 \leq i_{1}<i_{2}<\cdots<i_{a} \leq k} \prod_{s=1}^{a}\left[\left(\underline{\Lambda} \mid \underline{h}_{i_{s}}\right)+(a-s) \alpha_{0}\right],
$$

with $a=2, \ldots, k$. We should stress that the fields $\widetilde{\mathcal{W}}_{a}(z)$ are not quasi-primary and therefore are not exactly proportional to the Casimir operators of the coset (except for the field $\widetilde{\mathcal{W}}_{2}=\mathcal{W}_{2}$, the stress tensor); nevertheless, the Casimir operators are linear combinations of the fields $\widetilde{\mathcal{W}}_{a}$ and their derivatives. For example, the third order Casimir field in the $\mathcal{W}_{k}$ algebra is given by:

$$
\mathcal{W}_{3}(z)=\widetilde{\mathcal{W}}_{3}(z)-\frac{k-2}{2} \alpha_{0} \partial_{z} \widetilde{\mathcal{W}}_{2}(z)
$$

The Coulomb gas parameters in Eq. (4.27) are given by:

$$
\alpha_{+}+\alpha_{-}=\alpha_{0}, \quad \alpha_{+} \alpha_{-}=-1 \quad \text { and } \quad\left(\alpha_{0}\right)^{2}=\frac{1}{p(p+1)} \quad \text { for } \quad p=k+1 .
$$

The vectors $\left\{\underline{h}_{i}\right\}_{i=1}^{k}$ form the standard over-complete basis for the $s u(k)$ root lattice; they satisfy:

$$
\sum_{i=1}^{k} \underline{h}_{i}=0
$$

and express the positive roots and the fundamental weights by

$$
\underline{\alpha}^{i}=\underline{h}_{i}-\underline{h}_{i+1}, \quad \underline{\Lambda}_{i}=\sum_{s=1}^{i} \underline{h}_{s}, \quad i=1, \ldots, k-1 ;
$$




\begin{tabular}{|c||c|c|c|c|}
\hline Label & Field & Dimension $\Delta$ & $\mathbb{Z}_{3}$-charge $p$ & $\mathcal{W}_{3}$ charge \\
\hline \hline$\underline{\Lambda}_{0}+\underline{\Lambda}_{0}$ & 1 & 0 & 0 & 0 \\
\hline$\underline{\Lambda}_{0}+\underline{\Lambda}_{1}$ & $\sigma_{1}$ & $\frac{1}{15}$ & 1 & $-\frac{7}{675} \sqrt{5}$ \\
\hline$\underline{\Lambda}_{0}+\underline{\Lambda}_{2}$ & $\sigma_{2}$ & $\frac{1}{15}$ & 2 & $-\frac{2}{675} \sqrt{5}$ \\
\hline$\underline{\Lambda}_{1}+\underline{\Lambda}_{1}$ & $\psi_{1}$ & $\frac{2}{3}$ & 2 & $-\frac{22}{135} \sqrt{5}$ \\
\hline$\underline{\Lambda}_{1}+\underline{\Lambda}_{2}$ & $\varepsilon$ & $\frac{2}{5}$ & 0 & $-\frac{1}{25} \sqrt{5}$ \\
\hline$\underline{\Lambda}_{2}+\underline{\Lambda}_{2}$ & $\psi_{2}$ & $\frac{2}{3}$ & 1 & $\frac{4}{135} \sqrt{5}$ \\
\hline
\end{tabular}

Table 1: Primary fields of the $\mathbb{Z}_{3}$-parafermion theory and their quantum numbers, together with the eigenvalues of the $\widetilde{\mathcal{W}}_{2}$ and $\widetilde{\mathcal{W}}_{3}$ currents and of the $\mathbb{Z}_{3}$ charge.

therefore:

$$
\underline{h}_{i}=\underline{\Lambda}_{i}-\underline{\Lambda}_{i-1}, \quad i=1, \ldots, k, \quad\left(\underline{h}_{i} \mid \underline{h}_{j}\right)=\delta_{i j}-\frac{1}{k} ;
$$

(assuming $\underline{\Lambda}_{k} \equiv \underline{\Lambda}_{0} \equiv 0$ ). In particular, the Weyl vector has two different representations (the $\underline{h}_{i}$ are linearly dependent):

$$
\underline{\rho}=\sum_{i=1}^{k-1} \underline{\Lambda}_{i}=\frac{1}{2} \sum_{i=1}^{k}(k+1-2 i) \underline{h}_{i}=-\sum_{i=1}^{k} i \underline{h}_{i} .
$$

In order to compute the eigenvalues of the $\mathcal{W}_{k}$ generators we make the following identification of the weight:

$$
\underline{\Lambda} \equiv \alpha_{-}\left(\underline{\Lambda}_{\mu}+\underline{\Lambda}_{\nu}\right), \quad \text { where } \quad \alpha_{-}=-\frac{p}{\sqrt{p(p+1)}} .
$$

Using these formulas, we obtain the quantum numbers of the irreducible representations of the $\widetilde{\mathcal{W}}_{3}$ and $\widetilde{\mathcal{W}}_{4}$ algebras, which are listed in the Tables 1 and 2 , respectively.

Remark. In general, the Fateev-Lukyanov weights $\beta$ [15] are expressed as $\beta=$ $\alpha_{+} \underline{\Lambda}+\alpha_{-} \underline{\Lambda}^{\prime}$ where $\underline{\Lambda}$ and $\underline{\Lambda}^{\prime}$ are level 1 and $2 s u(k)$ weights respectively. From the coset point of view, however, the irreducible representations are labeled by triples consisting of two level 1 weights $\underline{\Lambda}_{\alpha}, \underline{\Lambda}_{\beta}$ and one level 2 weight $\underline{\Lambda}$ (see Eq. (4.1)). According to Eq. (4.3) the level 2 weight $\underline{\Lambda}$ together with one of the level 1 weights determine the entire triple since the remaining level 1 weight is fixed by charge conservation. It does not matter which of the level 1 weights we choose, so we characterize the triple by the second and the third weight $\left(\underline{\Lambda}_{\beta}, \underline{\Lambda}\right)$. In our case $(p=k+1)$, we can use the symmetries (4.4) of the pairs $\left(\underline{\Lambda}_{\beta}, \underline{\Lambda}\right)$ to put the levelone weight to zero as in Eq. (4.5); furthermore, we can always write a level-two $s u(k)$ weight as a sum of two fundamental weights. In conclusion, the FateevLukyanov weights are given by (4.31) (up to the factor $\sqrt{2}$ for any $a$ adjusting the normalization). 


\begin{tabular}{|l||c|c|c|}
\hline$\underline{\Lambda}_{\mu}+\underline{\Lambda}_{\nu}$ & $\Delta\left(\underline{\Lambda}_{\mu}+\underline{\Lambda}_{\nu}\right)$ & $\mathcal{W}_{3}\left(\underline{\Lambda}_{\mu}+\underline{\Lambda}_{\nu}\right)$ & $\mathcal{W}_{4}\left(\underline{\Lambda}_{\mu}+\underline{\Lambda}_{\nu}\right)$ \\
\hline \hline$\underline{\Lambda}_{0}+\underline{\Lambda}_{0}$ & 0 & 0 & 0 \\
\hline$\underline{\Lambda}_{0}+\underline{\Lambda}_{1}$ & $\frac{1}{16}$ & $-\frac{1}{160} \sqrt{30}$ & $-\frac{3}{1280} \sqrt{30}$ \\
\hline$\underline{\Lambda}_{0}+\underline{\Lambda}_{2}$ & $\frac{1}{12}$ & $-\frac{1}{180} \sqrt{30}$ & $\frac{7}{1440} \sqrt{30}$ \\
\hline$\underline{\Lambda}_{0}+\underline{\Lambda}_{3}$ & $\frac{1}{16}$ & $-\frac{1}{480} \sqrt{30}$ & $-\frac{7}{3840} \sqrt{30}$ \\
\hline$\underline{\Lambda}_{1}+\underline{\Lambda}_{1}$ & $\frac{3}{4}$ & $-\frac{7}{60} \sqrt{30}$ & $-\frac{7}{160} \sqrt{30}$ \\
\hline$\underline{\Lambda}_{1}+\underline{\Lambda}_{2}$ & $\frac{9}{16}$ & $-\frac{29}{480} \sqrt{30}$ & $-\frac{247}{3840} \sqrt{30}$ \\
\hline$\underline{\Lambda}_{1}+\underline{\Lambda}_{3}$ & $\frac{1}{3}$ & $-\frac{1}{45} \sqrt{30}$ & $-\frac{1}{90} \sqrt{30}$ \\
\hline$\underline{\Lambda}_{2}+\underline{\Lambda}_{2}$ & 1 & $-\frac{1}{15} \sqrt{30}$ & $-\frac{1}{15} \sqrt{30}$ \\
\hline$\underline{\Lambda}_{2}+\underline{\Lambda}_{3}$ & $\frac{9}{16}$ & $-\frac{7}{480} \sqrt{30}$ & $-\frac{3}{256} \sqrt{30}$ \\
\hline$\underline{\Lambda}_{3}+\underline{\Lambda}_{3}$ & $\frac{3}{4}$ & $\frac{1}{60} \sqrt{30}$ & $-\frac{1}{160} \sqrt{30}$ \\
\hline
\end{tabular}

Table 2: $\mathbb{Z}_{4}$-parafermion theory: labels of irreducible representations and eigenvalues of the $\widetilde{\mathcal{W}}_{2}, \widetilde{\mathcal{W}}_{3}$ and $\widetilde{\mathcal{W}}_{4}$ currents.

\section{Example: the $\mathbb{Z}_{3}$-parafermion Hall state}

We now spell out the coset construction of Section 4 in the case of $k=3$ (the analysis of the Pfaffian state $(k=2)$ can be found in Ref.[16]). Consider the lattice model of Section 3 for $k=3, M=1$ : the coset (1.2) coincides in this case with the $\mathbb{Z}_{3}$ Potts model, with $c_{\mathrm{PF}}=4 / 5$, which is also the well-known third minimal model of the Virasoro algebra; this model possesses six sectors, corresponding to the irreducible representations of the $\mathcal{W}_{3}$ algebra. On the other hand, the complete theory $\mathrm{PF}_{3} \oplus \widehat{u(1)}$ for the parafermion Hall state has ten sectors (this is the value of the topological order (4.17)). Let us discuss both theories in sequence.

The labels $\left(\underline{\Lambda}_{\mu}+\underline{\Lambda}_{\rho}\right)$ of the six parafermion primary fields given by the coset construction, their conformal dimensions $\Delta\left(\underline{\Lambda}_{\mu}+\underline{\Lambda}_{\rho}\right)$, the $\mathbb{Z}_{3}$ charges $p$ and the $\mathcal{W}_{3}$ charges are given in Table 1 . We can label the six sectors by an integer $\lambda$, $-2 \leq \lambda \leq 3$, related to the pair $(\mu, \rho)(0 \leq \mu, \rho \leq 2)$ of Eq. (4.6) as follows: $0=(0,0), 1=(1,0)=(0,1), \quad-1=(2,0)=(0,2), \quad 2=(1,1), \quad-2=(2,2)$, $3=(1,2)$. The conformal dimensions $\Delta_{\lambda}$ are given by:

$$
\Delta_{0}=0, \quad \Delta_{ \pm 1}=\frac{1}{15}, \quad \Delta_{ \pm 2}=\frac{2}{3}, \quad \Delta_{3}=\frac{2}{5} .
$$

The six coset characters (4.14) for $k=3$ (hereafter also called $c h_{\lambda}$ ) are:

$$
\begin{gathered}
c h_{2 l}(\tau)=\mathrm{Ch}_{0, l}(\tau)=q^{-\frac{1}{30}} \sum_{n_{1}+2 n_{2}=l \bmod 3,} \frac{q^{\frac{2}{3}\left(n_{1}^{2}+n_{2} n_{2}+n_{2}^{2}\right)}}{(q)_{n_{1}}(q)_{n_{2}}}, \quad l=0, \pm 1(\bmod 3), \\
\operatorname{ch}_{2 l-1}(\tau)=\mathrm{Ch}_{l+1, l+1}(\tau)=q^{\frac{1}{30}} \sum_{n_{1}+2 n_{2}=0 \bmod 3} \frac{q^{\frac{2}{3}\left(n_{1}^{2}+n_{2} n_{2}+n_{2}^{2}\right)-\frac{2 n_{l+1}+n_{2-l}}{3}}}{(q)_{n_{1}}(q)_{n_{2}}}, \quad l=0,1,
\end{gathered}
$$




$$
\operatorname{ch}_{3}(\tau)=\mathrm{Ch}_{1,2}(\tau)=q^{\frac{1}{30}} \sum_{n_{1}+2 n_{2}=2 \bmod 3} \frac{q^{\frac{1}{3}\left[2 n_{1}\left(n_{1}-1\right)+2 n_{1} n_{2}+n_{2}\left(2 n_{2}-1\right)\right]}}{(q)_{n_{1}}(q)_{n_{2}}} .
$$

These match the Rocha-Caridi characters $\chi_{\Delta}$ of the $c=4 / 5$ Virasoro minimal model 30]:

$$
\begin{aligned}
\mathrm{Ch}_{0,0} & =\chi_{0}+\chi_{3}, \\
\mathrm{Ch}_{0,1}=\mathrm{Ch}_{0,2} & =\chi_{2 / 3} \\
\mathrm{Ch}_{1,0}=\mathrm{Ch}_{2,0} & =\chi_{1 / 15} \\
\mathrm{Ch}_{1,2} & =\chi_{2 / 5}+\chi_{7 / 5} .
\end{aligned}
$$

The Potts-model partition function on the torus is given by the sum of the absolute value squared of these characters; these results verify that the symmetry algebra of this model is Virasoro extended by the $\mathcal{W}_{3}$ algebra 15.

The fusion rules of the primary fields in the theory are derived with the help of Section 4.4 and are listed in Table 3 (See Table 1 for the identification of the fields with their coset labels). Using these rules, it was found that the number of conformal blocks produced in the fusion of $3 \mathrm{~m}$ spin fields $\sigma_{1}$ is equal to the Fibonacci number $F_{3 m-2}[\mathbb{8}]$. Here we are interested in checking the assignment of the $\mathbb{Z}_{3}$ parafermion "charge" $p$ and its interplay with the fractional part of the physical charge $(\widehat{u(1)}$ part) in the full theory discussed hereafter.

The $\mathbb{Z}_{3}$-parafermion Hall state is characterized by the chiral algebra $\mathcal{A}_{3}$ defined by (4.8), the filling fraction $\nu_{3}=3 / 5$ and the central charge $c=9 / 5$; its ten sectors are described by the following characters:

$$
\begin{aligned}
\chi_{m \mu}(\tau, \zeta)= & \sum_{l=-1}^{1} K_{m+5 l}(\tau, 3 \zeta ; 15) c h_{m+3 \mu+2 l}(\tau) \\
& m=0, \pm 1, \pm 2, \quad \mu=0,1, \quad\left(c h_{\lambda}(\tau)=c h_{\lambda+6}(\tau)\right) .
\end{aligned}
$$

which are obtained by combining the charged $(K)$ and neutral $(c h)$ characters (see Eqs. (4.9) and (5.2)) according to the $\mathbb{Z}_{3}$ parity rule (4.11).

The minimal charges $Q_{m \mu}$ and minimal conformal dimensions $\Delta_{m \mu}$ of Hall edge excitations in the sectors (5.4) are:

$$
\begin{aligned}
& Q_{m, \mu}=\frac{m}{5}, \quad m=0, \pm 1, \pm 2, \quad \mu=0,1 \\
& \Delta_{m, 0}=\frac{1}{10}\left(\begin{array}{c}
|m|+1 \\
2
\end{array}\right), \quad \Delta_{0,1}=\frac{2}{5}=2 \Delta_{ \pm 2,1}, \quad \Delta_{ \pm 1,1}=\frac{7}{10}
\end{aligned}
$$

Quantum Hall states in the geometry of an annulus can be characterized by a (real) partition function which is (weakly) modular invariant, as described in Ref.[22]; this quantity completely accounts for the edge excitations on the two edges, 


$$
\begin{array}{lll}
\sigma_{1} \cdot \sigma_{1}=\sigma_{2}+\psi_{1} & \sigma_{2} \cdot \sigma_{2}=\sigma_{1}+\psi_{2} & \psi_{1} \cdot \psi_{1}=\psi_{2} \\
\sigma_{1} \cdot \sigma_{2}=1+\varepsilon & \sigma_{2} \cdot \psi_{1}=\sigma_{1} & \psi_{1} \cdot \varepsilon=\sigma_{2} \\
\sigma_{1} \cdot \psi_{1}=\varepsilon & \sigma_{2} \cdot \varepsilon=\sigma_{2}+\psi_{1} & \psi_{1} \cdot \psi_{2}=1 \\
\sigma_{1} \cdot \varepsilon=\sigma_{1}+\psi_{2} & \sigma_{2} \cdot \psi_{2}=\varepsilon & \varepsilon \cdot \varepsilon=1+\varepsilon \\
\sigma_{1} \cdot \psi_{2}=\sigma_{2} & \psi_{2} \cdot \psi_{2}=\psi_{1} & \varepsilon \cdot \psi_{2}=\sigma_{1}
\end{array}
$$

Table 3: Fusion rules of the $\mathbb{Z}_{3}$-parafermion theory.

which carry opposite chirality and satisfy some global conditions, such as total integer charge and half-integer spin. The partition function for the $\mathbb{Z}_{3}$-parafermion Hall state is given by the diagonal modular invariant:

$$
Z(\tau, \zeta)=\sum_{m, \mu}\left|\widetilde{\chi}_{m \mu}\right|^{2}
$$

(this form obviously extends to higher $k$ values). In this equation, the characters $\tilde{\chi}$ are given by the complete characters (5.4) times a convenient real factor [22]:

$\tilde{\chi}_{m \mu}=\exp \left(-\frac{\pi}{5} \frac{(\operatorname{Im} \zeta)^{2}}{\operatorname{Im} \tau}\right) \chi_{m \mu}$. The invariance of the partition function under the modular transformations $T^{2}:(\tau, \zeta) \rightarrow(\tau+2, \zeta)$ and $S:(\tau, \zeta) \rightarrow(-1 / \tau, \zeta / \tau)$ holds because the characters (5.4) transform linearly by unitary matrices. In particular, the $S$ modular inversion is represented by the matrix (up to an overall phase [22]):

$$
S_{m \mu, m^{\prime} \mu^{\prime}}=\frac{\sqrt{3-\delta}}{5}(-1)^{\mu \mu^{\prime}+m \mu^{\prime}+m^{\prime} \mu} \mathrm{e}^{i \frac{\pi}{5} m m^{\prime}} \delta^{P_{\mu+m+\mu^{\prime}+m^{\prime}}},
$$

with $m, m^{\prime}=0, \pm 1, \pm 2, \mu, \mu^{\prime}=0,1, \mathrm{e}^{i \pi / 5}=(\delta+i \sqrt{3-\delta}) / 2$ and $P_{\lambda}=\left(1-(-1)^{\lambda}\right) / 2$.

The matrix elements of $S$ determine the so-called quantum dimension $d_{q}$ of the representation $(m, \mu)$, which is:

$$
d_{q}(m, \mu)=\frac{S_{m \mu, 00}}{S_{00,00}}=\left\{\begin{array}{lll}
1 & \text { for } \mu+m & \text { even }, \\
\delta & \text { for } \mu+m & \text { odd } .
\end{array}\right.
$$

The presence of a non-integer (in fact, irrational) quantum dimension $\delta$ signals the non-abelian statistics of the quasi-particle excitations in this Hall state (already discussed at the level of wave functions in Section 2.1). Indeed, some fusion rules yields more than one field in the r.h.s., as shown in Table 3 .

\section{Conclusions and a general framework}

In this paper, we have described the conformal theory of $\mathbb{Z}_{k}$-parafermion Hall states by a two-step procedure: first introducing a lattice current algebra theory corresponding to the same filling fraction, and then taking a coset projection. We have 
presented two abelian theories, with central charge $c=k$ and $c=2 k-1$, respectively. The $c=k$ one is useful to write wave functions which nicely describe the origin of the non-abelian statistics of quasi-particles as the effect of the projection. The $c=2 k-1$ theory accounts for a complete definition and description of all excitations of the parafermion Hall states, which are deduced from a highly symmetric choice of charge lattice followed by the coset construction.

In conclusion we would like to briefly outline a general program of defining and classifying conformal field theories suitable for describing quantum Hall states. Our objective here would be to directly characterize the outcome - without reference to the parent abelian theory - and find the lattice theories and coset models as special cases, in the same spirit as in Ref.[23]. We sum up the basic characteristics of the theory by a set of four postulates:

P1. Each Hall plateau and its excitations are described by a rational conformal field theory with a local chiral algebra $\mathcal{A}$ which contains a $\widehat{u(1)}$ "electric current" $J(z)$ and an odd (Fermi) electron field $\Psi_{e}$ of charge $\left(\underline{Q} \mid \underline{q}_{e}\right)=-1$ and minimal (in $\mathcal{A})$ half-integer dimension $\Delta_{e}=p+1 / 2$ (and its conjugate field $\Psi_{e}^{*}$ ).

P2. The neutral sub-algebra $\mathcal{A}_{0}$ of $\mathcal{A}$,

$$
\mathcal{A}_{0}=\widehat{u(1)} \otimes \mathcal{C}, \quad\left(\Phi_{e}(z) \in \mathcal{C} \quad \text { iff } \quad\left[J(z), \Phi_{e}(w)\right]=0\right)
$$

is bosonic. The electron is associated to a primary (with respect to $\mathcal{A}_{0}$ ) - field $\Psi_{e}$ satisfying the fusion rule:

$$
\Psi_{e}^{*} \cdot \Psi_{e} \in \mathcal{A}_{0}
$$

(Hence, it is a "simple current", see Section 5.1 of [23.)

P3. The electron field transforms under a tensor product representation of $\widehat{u(1)} \otimes$ $\mathcal{C}: \Psi_{e}(z)=Y\left(\left(\nu_{k}\right)^{-1 / 2}, z\right) \phi_{k}(z)$, such that:

$$
\phi_{k}\left(z_{1}\right) \cdots \phi_{k}\left(z_{k}\right) \in \mathcal{A}_{0}
$$

here, $k$ is a multiple of the numerator of the fractional part $\nu_{k}$ of the filling fraction $\left(0<\nu_{k}<1\right)$. Its conformal dimension splits correspondingly into a sum of two terms (cf. Eq.(3.9)):

$$
2 \Delta_{e}=\frac{1}{\nu_{k}}+2 \Delta_{0}
$$

In particular, for a Laughlin fluid, $k=1$ and $\phi_{1}\left(z_{1}\right)=1$. This postulate represents the $\mathbb{Z}_{k}$ selection rule in the general framework.

P4. The admissible excitations (or the superselection sectors) of the Hall state are described by the primary fields of $\mathcal{A}$ that are, in particular, relatively local to $\Psi_{e}$. (This is, in fact, part of the definition of a rational conformal field theory with chiral algebra $\mathcal{A}$.)

Here are some implications of these postulates: 
i) The rationality of the theory implies that the filling fraction and all conformal dimensions are rational numbers.

ii) The charge-statistics relation (which generalizes (1.4): odd and even square charges correspond to Fermi and Bose fields, respectively) is a consequence of P1 and P2. Indeed, (composite) Bose fields in $\mathcal{A}_{0}$ contain an even number of electron field factors and, hence, carry an even charge.

iii) If $\nu_{k}=n / d$ (where $n, d$ are natural numbers) then $k=\ln$ and the minimal (non zero) absolute value of the charge of an excitation is $1 /(l d)$ (cf. Section 5.1 of [23]).

iv) The characters of the superselection sectors of the conformal theory with chiral algebra $\mathcal{A}$ (obeying $\mathrm{P} 4$ ) span a representation of the subgroup $\Gamma_{2}$ of the modular group $S L(2, \mathbb{Z})$ generated by $S$ and $T^{2}$. If we adopt, in addition, the postulates (or, rather, observations) (S1) and (S3) of [23], saying that a Hall state is more stable the smaller is the Virasoro central charge and the topological order (i.e., the number of independent excitations), then the coset models considered in this paper are clearly favoured compared to the lattice theories describing the same filling fractions.

\section{Acknowledgements}

L. G. thanks C. Schweigert for fruitful discussions. L. G. thanks I.N.F.N., Firenze for hospitality; I. T. thanks the Institut des Hautes Etudes Scientifiques (IHES), Bures-sur-Yvette, for hospitality. L.G. and I.T. acknowledge partial support from the Bulgarian National Foundation for Scientific Research under contract F-828. A.C. acknowledges the partial support from the European Community network programme FMRX-CT96-0012.

\section{References}

[1] For a review see: R. A. Prange and S. M. Girvin, The Quantum Hall Effect, Springer Verlag, New York (1990); S. Das Sarma and A. Pinczuk, Perspectives in Quantum Hall effects, John Wiley (1997).

[2] For a review, see: X.-G. Wen, Int. J. Mod. Phys. 6 B (1992) 1711; Adv. in Phys. 44 (1995) 405.

[3] R. B. Laughlin, Phys. Rev. Lett. 50 (1983) 1395; for a review see: R. B. Laughlin, Elementary Theory: the Incompressible Quantum Fluid, in [1].

[4] L. Saminadayar, D. C. Glattli, Y. Jin and B. Etienne, Phys. Rev. Lett. 79 (1997) 2526; R. de Picciotto, M. Reznikov, M. Heiblum, V. Umansky, G. Bunin and D. Mahalu, Nature 389 (1997) 162. 
[5] G. Moore and N. Read, Nucl. Phys. B 360 (1991) 362; M. Milovanović and N. Read, Phys. Rev. B53, 13559 (1996); N. Read and R. H. Rezayi, Phys. Rev. B 56 (1996) 16864.

[6] F.D.M. Haldane and E.H. Rezayi, Phys. Rev. Lett. 60 (1988) 956; 60 (1988) 1886; E.H. Rezayi, unpublished.

[7] R. H. Morf, Phys. Rev. Lett. 80 (1998) 1505; E. H. Rezayi and F. D. M. Haldane, preprint cond-mat/9906137.

[8] N. Read and E. Rezayi, Phys. Rev. B 59 (1998) 8084.

[9] A.B. Zamolodchikov and V.A. Fateev, Sov. Phys. JETP 62 (1985) 215, Nucl. Phys. B 280(1987) 644; D. Gepner and Z. Qiu, Nucl. Phys. B285 (1987) 423.

[10] W. Pan et al., Phys. Rev. Lett. 83 (1999) 3530.

[11] V. Gurarie and E. Rezayi, Phys. Rev. B61 (2000) 5473.

[12] E. Ardonne, P. Bouwknegt and K. Schoutens, Non-abelian quantum Hall states - exclusion statistics, K-matrices and duality, preprint cond-mat/0004084.

[13] A. Cappelli, C. A. Trugenberger and G. R. Zemba, Nucl. Phys. B 396 (1993) 465, Nucl. Phys. B 448 (1995) 470; Cappelli and G. R. Zemba, Nucl. Phys. B 540 (1999) 610; S. Iso, D. Karabali and B. Sakita, Nucl. Phys. B 388 (1992) 700; for a review, see: A. Cappelli, C. A. Trugenberger and G. R. Zemba, Proc. of the Int. Workshop on Low Dimensional Field Theory, Telluride, Int. J. Mod. Phys. A 12 (1997) 1101-1111.

[14] A. Cappelli, C. A. Trugenberger and G. R. Zemba, Nucl. Phys. B 448 (1995) 470

[15] V. A. Fateev and A. B. Zamolodchikov, Nucl. Phys. B280 (1987) 644; V. A. Fateev and S. L. Lukyanov, Int. J. Mod. Phys. A3 (1988) 507, A7 (1992) 853; for a review, see: P. Bouwknegt and K. Schoutens, Phys. Rept. 223 (1993) $183-276$.

[16] A. Cappelli, L. S. Georgiev and I. T. Todorov, Commun. Math. Phys. 205 (1999) 657.

[17] M. Greiter, X.-G. Wen and F. Wilczek, Nucl. Phys. B374 (1992) 567; Phys. Rev. Lett. 66 (1991) 3205.

[18] A. Cappelli, L. S. Georgiev and I. T. Todorov, Coset Construction of Parafermionic Hall States, preprint ESI/828, Proc. of Supersymmetries and Quantum Symmetries, SQS'99, July 1999, Dubna, E. Ivanov, S. Krivonos and A. Pashev Eds., JINR (2000) 235. 
[19] P. Griffin and D. Nemeschansky, Nucl. Phys. B323 (1989) 545.

[20] P. Goddard, A. Kent and D. Olive, Commun. Math. Phys. 103 (1986) 105.

[21] J. Fröhlich and E. Thiran, J. Stat. Phys. 76 (1994) 209; J. Fröhlich, U. M. Studer and E. Thiran, J. Stat. Phys. 86 (1997) 821;J. Fröhlich, T. Kerler, U. M. Studer and E. Thiran, Nucl. Phys. B 453 (1995) 670.

[22] A. Cappelli and G.R. Zemba, Nucl. Phys. B490 (1997) 595.

[23] J. Fröhlich, B. Pedrini, C. Schweigert and J. Walcher, Universality in Quantum Hall Systems: Coset Construction of Incompressible States, preprint condmat/0002330.

[24] E. Fradkin, C. Nayak and K. Schoutens, Nucl. Phys. B546 (1999) 711; X.-G. Wen, Phys. Rev. B60 (1999) 8827.

[25] N. Read and D. Green, Phys. Rev. B61 (2000) 10267

[26] C. Nayak and F. Wilczek, Nucl. Phys. B479 (1996) 525.

[27] V.G. Kac and I.T. Todorov, Commun. Math. Phys. 190 (1997) 57.

[28] J. Fuchs, A.N. Schellekens and C. Schweigert, Nucl. Phys. B461 (1996) 39.

[29] A. Schilling, Nucl. Phys. B459, 393 (1996), Nucl. Phys. B467, 247 (1996), and references therein.

[30] B. L. Feigin and D. B. Fuchs, Funct. Anal. Appl. 17 (1983) 241; A. RochaCaridi, in Vertex operators in mathematics and physics, J. Lepowski et al. eds., Springer, New York (1985). 